\title{
比较两种丝素膜的结构、热分解机理 与热力学参数
}

刘其春 ${ }^{1,2}$, 王昉 ${ }^{*}$, 李莹莹 ${ }^{1,2}$, 于海洋 $^{1,2}$, 马青玉 ${ }^{3}$, 顾正桂 ${ }^{2 *}$

1. 南京师范大学分析测试中心, 南京 210023

2. 南京师范大学化学与材料科学学院, 南京 210023

3. 南京师范大学物理科学与技术学院, 南京 210023

*通讯作者, E-mail: wangfang@njnu.edu.cn; guzhenggui@njnu.edu.cn

收稿日期: 2018-12-23; 接受日期: 2019-01-21; 网络版发表日期: 2019-03-18

国家自然科学基金(编号: 11474166)和江苏省高校自然科学基金(编号: 15kJB150018)资助项目

摘要本文利用氯化钙-甲酸体系制备了来源于中国和泰国家蚕的两种丝素膜. 通过扫描电子显微镜、傅里叶 红外光谱和 $\mathrm{X}$ 射线衍射以及热重分析技术，分别表征了膜材料的形貌、分子构象、热稳定性以及热分解机理，获 得了动力学参数(活化能 $E$ 和指前因子 $A$ ) 和热力学参数 $\left(\Delta H^{\neq} 、 \Delta G^{\neq}\right.$和 $\Delta S^{\neq}$), 并对其进行了比较研究. 结果表明, $1.5 \mathrm{wt} \%$ 浓度体系下的丝素膜结构主要呈现 $\beta$-折叠， $3.0 \mathrm{wt} \%$ 浓度体系下的膜结构以无规线团或 $\alpha$-螺旋为主; $3.0 \mathrm{wt} \%$ 浓度下的膜在不同升温速率下的分解温度、活化能和活化焓均低于 $1.5 \mathrm{wt} \%$ 浓度下的，后者的热稳定性 优于前者; 相同浓度体系下, 泰国丝素膜的 $\beta$-折叠含量略高于中国丝素膜, 具有更好的热稳定性; 通过Achar微分 法和Coats-Redfern积分法计算发现，两种丝素膜在464 594 K的热分解过程都遵循二维扩散机理.

关键词丝素, 分子构象, 热分解机理, 热力学, 热分析

\section{1 引言}

在自然界众多的天然高分子材料中，人们对䗞丝 格外关注，因为其除了具有吸湿性和鲜亮的光泽，被 人们用作高档的纺织材料之外，它还具有良好的力学 性能、生物相容性以及生物可降解性等，可以应用于 食品、医药、生物技术和日用精细化工等领域 ${ }^{[1 \sim 5]}$.

天然蚕丝经脱胶、溶解、冷冻干燥、自然干燥或 通过静电纺丝等工序后，可以制成再生丝素材料 ${ }^{[6-9]}$. 但是，再生丝素材料性能的优劣，在很大程度上受到
其制备工艺的影响. 制备方法可由无机盐水溶液 ${ }^{[10]}$ 、 磷酸溶液 ${ }^{[11]}$ 或离子液体制备 ${ }^{[12]}$, 然而, 这样获得的丝 素蛋白溶液稳定性不够，且存在蛋白析出或制备周期 长的缺点 ${ }^{[13]}$. 本课题组在前期制备出水溶性丝素膜 ${ }^{[14]}$. 利用氯化钙-甲酸体系进行制备 ${ }^{[15]}$, 不仅能有效解决上 述难题, 还能够获得力学性能优良且不溶于水和具有 纳米纤维结构的丝素膜 ${ }^{[16,17]}$.

由于受到生长环境和地域的影响, 同属于Bombyx mori科系的不同地域的蚕丝性能结构会有差异. 例如, 同吃桑叶的来源于中国家鉵丝多数呈现白色，来源于

引用格式: Liu Q, Wang F, Li Y, Yu H, Ma Q, Gu Z. Comparative studies of structure, thermal decomposition mechanism and thermodynamic parameters of two kinds of silk fibroin films. Sci Sin Chim, 2019, 49: 1014-1029, doi: 10.1360/N032018-00265 
泰国的家蚌丝呈金黄色，它们的玻璃化转变温度和力 学性能也不同 ${ }^{[14]}$.

对于再生丝素材料的分析研究与表征手段有多 种, 其中, 热分析技术是最有效的分析方法之一. 通过 它可以评估材料的热稳定性，包括热分解温度、热降 解速率和老化时间等特性 ${ }^{[18 \sim 21]}$, 同时也可以进行热力 学和热分解动力学研究 ${ }^{[22 ~ 24]}$. 本课题组 ${ }^{[25]}$ 曾经制备了 微孔聚乳酸支架材料, 利用Kissinger、Ozawa-Doyle和 Vyazovkin方程进行热分解动力学计算, 并推算氮气环 境下材料的降解时间和使用寿命. Sreeram 等 ${ }^{[26]}$ 通过等 温热重量分析法对聚对苯乙烯共轭聚合物膜进行反应 动力学研究, 他们发现聚对苯氯乙烯脱氯化氢作用的 活化能显著低于聚氯乙烯发生脱氯化氢作用所需的活 化能, 后者可以在更低的温度下发生脱除氯化氢反应.

本文利用氯化钙-甲酸体系分别制备了来源于中 国(MSF)和泰国家蚕(TSF)的两种丝素膜，利用扫描电 子显微镜(SEM)观察了膜材料的形貌, 傅里叶红外光 谱(FTIR)和X射线衍射(XRD)技术表征膜材料的分子 构象, 热重分析(TG)测定了膜材料的热稳定性和热分 解特性, 并采用Kissinger、Ozawa和Vyazovkin数学模 型计算了热分解过程的动力学参数, 探讨了其热稳定 性机理和氯化钻浓度对膜材料热分解过程的热力学影 响. 同时，利用Achar微分法和Coats-Redfern积分法进 行热分解最可几机理函数的判定，并对两种来源的丝 素膜进行了比较研究. 本研究提供的丝素膜的热变 性、稳定性以及热分解机理和热力学参数等有用信 息，可以为丝素蛋白材料及其复合材料的合成研究以 及实际生产工艺路线的选择提供帮助，对促进绿色天 然材料的实际利用, 具有重要的理论与实践意义.

\section{2 实验部分}

\section{1 化学试剂及仪器}

材料与试剂: 中国家蚕丝(丹东七月贸易有限公 司); 泰国家虽丝(泰国Nakornratchasima皇后养蚕中心); 无水氯化钙 $(96.00 \%, \mathrm{AR}$ 纯度), 甲酸 $(88.00 \%, \mathrm{AR}$ 纯度), 丙酮 $(99.50 \%, A R$ 纯度), 广东汕头市西陇化工厂.

仪器: (1) 将材料放置在溅射仪内, 设置电流为 $20 \mathrm{~mA}$, 每个面镀三次, 每次 $10 \mathrm{~s}$; SEM的工作电压为 $10 \mathrm{kV}$, 工作距离为 $15 \mathrm{~mm}$, 镀好膜的样品放入日本 JEOL公司的JSM-7600F的扫描电子显微镜的样品腔
内，进行形貌观察；(2) 将样品直接放在美国Thermo Nicolet公司NEXUS-670型FTIR光谱仪的Ge晶体上, 旋 转OMNI采样器固定钮, 压住样品, 利用单反射ATROMNI采样器进行信号收集, 分辨率为 $4 \mathrm{~cm}^{-1}$, 扫描次 数为 32 次, 光谱范围为 $1750 \sim 1450 \mathrm{~cm}^{-1}$ 进行红外光谱 测定; (3) 利用日本理学公司生产的 $\mathrm{D} / \mathrm{max} 2500 / \mathrm{pc}$ 型 $\mathrm{X}$ 射线衍射仪对样品进行测定, 使用 $\mathrm{Cu} \mathrm{K \alpha} \alpha$ 辐射, 管压 为 $40 \mathrm{kV}$ 、管流为 $200 \mathrm{~mA}$, 衍射角 $2 \theta$ 范围为 $5^{\circ} \sim 50^{\circ}$, 扫 描速率为 $5^{\circ} \mathrm{min}^{-1}$; (4) 利用美国PerkinElmer公司生产 的Pyris $1 \mathrm{TGA}$ 热重分析仪进行样品测定, 氮气气氛作 为保护气, 氮气流速为 $50 \mathrm{~mL} \mathrm{~min}^{-1}$ 的情况下, 升温速 率分别为 $2 、 5 、 10$ 和 $15 \mathrm{~K} \mathrm{~min}^{-1}$, 温度范围为室温 $\sim 900.00 \mathrm{~K}$.

\section{2 丝素膜的制备}

称取一定等质量的中国家蚕脱胶蚕丝，分别置于 $1.50 \mathrm{wt} \%$ 和 $3.00 \mathrm{wt} \%$ 浓度的氯化钙-甲酸溶液中溶解, 配制成8.00 wt\%的丝素蛋白溶液. 经过过滤、浇铸、 去除溶剂、漂洗、风干，获得氯化钙浓度 $1.50 \mathrm{wt} \%$ 和 $3.00 \mathrm{wt} \%$ 的中国家䖯丝素膜(MSF-1.5 和MSF-3.0). 用 上述同样方法制得泰国家蚕丝素膜( TSF-1.5 和 TSF-3.0).

\section{3 结果与讨论}

\section{1 丝素膜的表观形貌}

图1上层为中国家蚕丝素膜和泰国家蚕丝素膜的 扫描电子显微镜图, 下层是对上层红色方框某个区域 再放大(下层为放大 50000 倍的SEM) 的形貌图. 可以看 出, MSF在氯化钻溶液浓度为 $1.5 \mathrm{wt} \%$ 时(图 1 (a)), 膜表 面的纤维形成了三维的多孔结构. 50000倍下SEM观 察, 可以看到均匀、致密的微纤维结构; 当浓度增大 到3 wt\%时(图1(b)), MSF的丝素纤维三维结构变得润 滑, 在高倍下呈现紧密团状结构; 而泰虫丝素膜TSF1.5 (图1(c))表面的纤维出现膨润条状结构, 这可能是 由于氯化钲首先渗透到纤维结构的内部，与丝素中酪 氨酸残基酚羟基邻位上的氢发生交换，并破坏了链间 的共价键 ${ }^{[15]}$; TSF-3.0表面平滑而突兀(图1(d)), 50000 倍下SEM观察, 呈现细小纤维颗粒状, 说明丝素表面的 纤维结构进一步膨润、溶失. 有文献指出 ${ }^{[8]}$, 氯化钲浓 度的增大会导致丝蛋白酚羟基邻位上的氢交换量增 


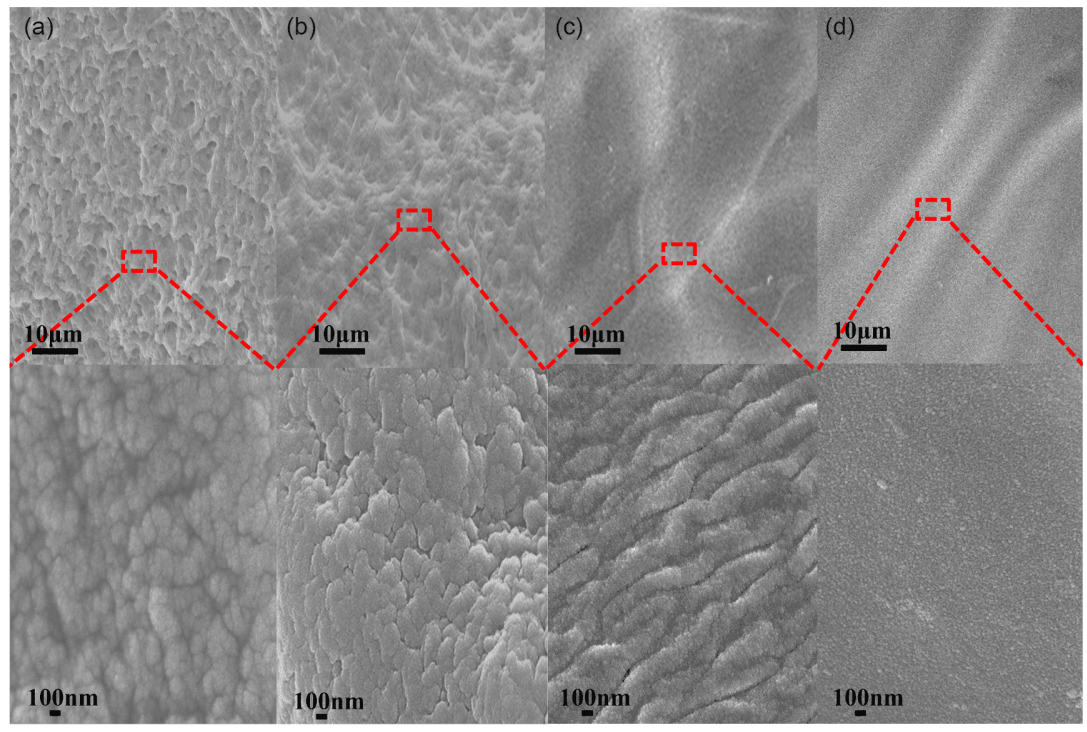

图 1 丝素膜MSF-1.5 (a)、MSF-3.0 (b)、TSF-1.5 (c)和TSF-3.0 (d)的SEM形貌图. 上图标尺: $10 \mu \mathrm{m}$, 下图标尺: $100 \mathrm{~nm}$ (网络版 彩图)

Figure 1 SEM images of (a) MSF-1.5, (b) MSF-3.0, (c) TSF-1.5, (d) TSF-3.0 (top scale bar: $10 \mu \mathrm{m}$, bottom scale bar: $100 \mathrm{~nm}$ ) (color online).

加, 纤维结构膨润, 直至发生溶解.

\section{2 丝素膜的结构}

图2是中国丝素膜MSF-1.5和MSF-3.0的红外光谱 图, 从图中可以看出, MSF-1.5在酰胺I区的红外特征峰 都出现在 $1625 \mathrm{~cm}^{-1}$, 酰胺II的红外特征峰都在 $1523 \mathrm{~cm}^{-1}$. 这表明MSF-1.5 的结构主要是 $\beta$-折叠结 构 ${ }^{[27]}$, 并且在 $1645 \mathrm{~cm}^{-1}$ 还有一个小肩峰, 说明MSF1.5 结构中还含有一定量的 $\beta$-turn结构 ${ }^{[28]}$; 当溶液中氯 化钙浓度达到 $3.00 \mathrm{wt} \%$ 时, 从图中可以看出酰胺 $\mathrm{I}$ 区只 出现一个红外吸收峰 $\left(1645 \mathrm{~cm}^{-1}\right)$, 酰胺II区的峰出现 在 $1545 \mathrm{~cm}^{-1}$, 这说明当氯化钙的浓度达到 $3.00 \mathrm{wt} \%$ 时, 丝素蛋白结构中主要以无规线团结构为主. 有文献指 出 ${ }^{[29,30]}$, 钲离子会渗透到丝素纤维内部, 破坏蛋白分 子内部的氢键和范德华力等次价键. 同时，钻离子与 丝素蛋白分子链上的丝氨酸、酪氨酸和侧链羟基发生 配位, 使得红外吸收峰位向高波数移动, 蛋白结构由 $\beta$ 折叠变成以无规线团为主. 因此可以推断, MSF-1.5中 主要以 $\beta$ 折叠为主, MSF-3.0中主要以无规卷曲为主. 表1为中国家䖯丝和泰国家贵丝在酰胺I和酰胺II区的 红外特征峰归属. 从表中可以看出在相同浓度制备下 的丝素膜在酰胺I区的特征峰归属是相同的 $(1.5 \%$ : $1625 \mathrm{~cm}^{-1} ; 3.0 \%$ : $\left.1645 \mathrm{~cm}^{-1}\right)$; 但在酰胺II区有着显著

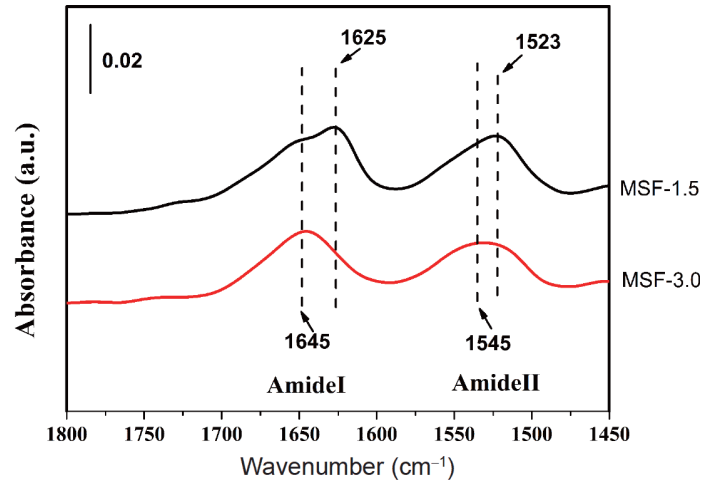

图 2 MSF-1.5 和MSF-3.0的红外光谱图(网络版彩图)

Figure 2 Fourier transform infrared (FTIR) spectra of MSF-1.5 and MSF-3.0 (color online).

的差异: MSF-1.5 和MSF-3.0的特征峰分别为 1523 和 $1545 \mathrm{~cm}^{-1}$; TSF-1.5 和TSF-3.0特征峰分别为 1522 和 $1534 \mathrm{~cm}^{-1}$. 因此, 两种丝素膜都随着氯化钲浓度的增 加, 其对应的红外特征峰往高波数偏移. 这些结果表 明, 氯化钻浓度对这两种丝素具有相同的影响.

一般认为，丝素蛋白有两种结晶结构，即 silk I和 silk II结构. silk I结构的主要构象是 $\alpha$-螺旋和无规线团, 其对应的衍射角 $2 \theta$ 在 $12.2^{\circ} 、 28.2^{\circ} 、 24.7^{\circ}$ 和 $27.9^{\circ}$ 附近, silk II结构 (主要构象是 $\beta$-折叠) 的主要衍射峰位在 $18.9^{\circ} 、 20.7^{\circ}$ 和 $24.0^{\circ}$ 附近 ${ }^{[31,32]}$. 从XRD衍射图(图3)中 
表 1 两种丝素膜的红外归属 ${ }^{a)}$

Table 1 Attribution of infrared characteristic peak of two silk fibroin films ${ }^{\text {a) }}$

\begin{tabular}{ccccc}
\hline 样品 & 来源 & 氯化钲浓度 $(\%)$ & 酰胺I区 $\left(\mathrm{cm}^{-1}\right)$ & 酰胺II区 $\left(\mathrm{cm}^{-1}\right)$ \\
\hline MSF-1.5 & 中国家蚻 & 1.5 & 1625 & 1523 \\
TSF-1.5 & 泰国家蚕 & 1.5 & 1625 & 1522 \\
MSF-3.0 & 中国家蚻 & 3.0 & 1645 & 1545 \\
TSF-3.0 & 泰国家虫 & 3.0 & 1645 & 1534 \\
\hline
\end{tabular}

a) 以上红外实验为每个样品测试3次, 最大误差 $\Delta V \leq 2 \mathrm{~cm}^{-1}$

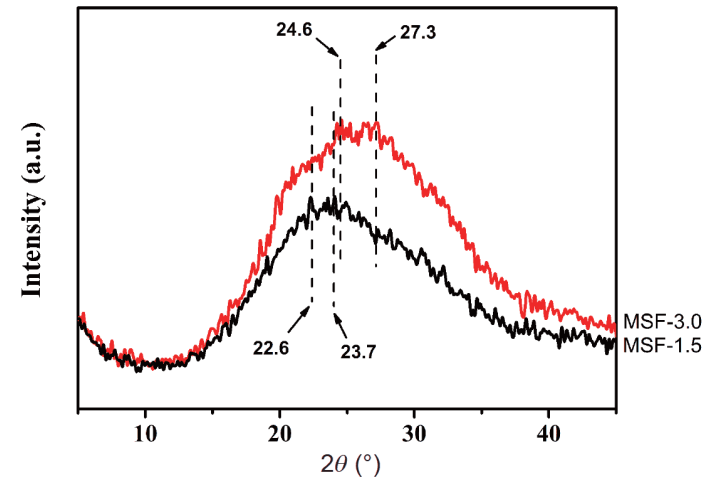

图 3 MSF-1.5 和MSF-3.0的X射线衍射图(网络版彩图) Figure 3 X-ray diffraction (XRD) patterns of MSF-1.5 and MSF-3.0 (color online).

可以看到, MSF-1.5在宽衍射峰中有两个小衍射峰, 分 别在 $22.6^{\circ}$ 和 $23.7^{\circ}$, 根据衍射峰位的判断, 这个丝素膜 中含有一定量的silk II结构 ( $\beta$-折叠结构); MSF-3.0的宽 衍射峰中也同样出现两个小衍射峰，但与MSF-1.5相 比，其衍射峰都向高位偏移，并且其对应的衍射峰位 分别在 $24.6^{\circ}$ 和 $27.3^{\circ}$, 这两处的衍射峰归属于silk I结 构. 由此可见, 浓度提高使得MSF-3.0内含有较多的silk I结构. 这一现象表明, 较高的氯化钲浓度可能会破坏 丝素蛋白分子链间的氢键，使其silk II向silk I结构转 变. 对丝素膜的XRD和FTIR谱图进行高斯函数拟合和 计算 $\beta$-折叠含量, 列于表 2 . 从表 2 的XRD计算值看出, 氯化钻浓度为 $1.5 \%$ 的丝素膜的 $\beta$-折叠含量分别为: 中 国丝素为 $48.46 \%$ ，泰国丝素为 $50.23 \%$; 氯化钻浓度为 $3.0 \%$ 的丝素膜的 $\beta$-折叠含量分别为: 中国丝素膜为 $21.84 \%$ ，泰国丝素膜为 $22.69 \%$. 说明氯化钲浓度使得 silk II结晶含量少于MSF-1.5. FTIR实验数据拟合计算 的结果与此结果一致(表2). 比较两种丝素膜中 $\beta$-折叠 含量发现, 在相同浓度制备下, MSF的 $\beta$-折叠含量略低 于TSF.

\section{3 丝素膜的热分解机理}

\subsection{1 丝素蛋白的热稳定性}

由图4可知，中国家虫丝素膜存在两个失重阶段: 第一阶段的失重台阶是在 $464 \mathrm{~K}$ 之前, 这主要是一些 自由水和低沸点化合物 ${ }^{[33]}$, 失重量约为 $1 \%$; 第二阶段 失重是从 $464 \mathrm{~K}$ 开始, 同时丝素膜在 TG曲线上达到最 大分解速率时所对应温度 $T_{\mathrm{p}}$, 即 DTG曲线上最大峰所 对应的峰温在 564 594 K之间, 并且在 $900.00 \mathrm{~K}$ 时总失 重量可达 $60 \%$ 左右，这一阶段可以认为是丝素膜中丝 素蛋白分子间侧链和主链遭到破坏引起的分解失 重 ${ }^{[34]}$. 从图4中可以看出, MSF-1.5 的起始分解温度 $\left(T_{\mathrm{on}-}\right.$ set) 比MSF-3.0高, 这可能是由于高浓度的销离子对丝 素蛋白分子间的氢键破坏程度更大, 使其silk II $(\beta$-折 叠)含量减小, 导致其分子链段更加无序化, 非晶化程 度增大, 从而使得膜的分解温度降低, 热稳定性下 降 $^{[35]}$. 在泰国丝素膜的实验中, 采用相同的实验条件 进行测定, 结果列于表 3. 其中, 泰国丝素膜TSF-1.5 的 $T_{\mathrm{p}}$ 和 $T_{\text {onset }}$ 分别为 570.53 和 $551.03 \mathrm{~K} ; \mathrm{TSF}-3.0$ 的 $T_{\mathrm{p}}$ 和 $T_{\text {onset }}$ 分别为 566.92 和 $545.81 \mathrm{~K}$. 另外, 以 $T_{\mathrm{p}}$ 为参照温度, 发现 该温度下MSF-1.5 和TSF-1.5 的残余量较多, 分别可达 $78.46 \%$ 和 $77.80 \%$, 当氯化钲浓度增加到 $3 \mathrm{wt} \%$ 时, 膜的 失重量会逐渐增加. 进一步说明了氯化钙浓度对两种 丝素膜的稳定性具有相同的影响, 其中, 泰丝素蛋白 膜有较好的热稳定特性.

\subsection{2 丝素膜的热分解动力学参数}

MSF-1.5、MSF-3.0、TSF-1.5和TSF-3.0的热分解 动力学基础数据列于表 4 . 从表 4 中可以看出, 丝素膜起 始分解温度和最大分解速率对应温度以及失重反应所 对应的特征温度均随着升温速率的提高向高温方向偏 移, 但不影响总失重量 ${ }^{[36,37]}$. 我们将对丝素膜第二阶段 
表 2 两种丝素膜的FTIR和XRD的拟合结果 ${ }^{\text {a) }}$

Table 2 Fitting results of two kinds of silk fibroin films by FTIR and XRD ${ }^{\text {a) }}$

\begin{tabular}{|c|c|c|c|c|}
\hline \multirow{2}{*}{ 样品 } & \multirow{2}{*}{ 来源 } & \multirow{2}{*}{ 氯化钻浓度 (\%) } & \multicolumn{2}{|c|}{$\beta$-折叠含量 $(\%)$} \\
\hline & & & XRD拟合计算 & FTIR拟合计算 \\
\hline MSF-1.5 & 中国家蚕 & 1.5 & 48.46 & 45.88 \\
\hline TSF-1.5 & 泰国家蚕 & 1.5 & 50.23 & 48.86 \\
\hline MSF-3.0 & 中国家虫 & 3.0 & 21.84 & 18.25 \\
\hline TSF-3.0 & 泰国家蚕 & 3.0 & 22.69 & 20.14 \\
\hline
\end{tabular}

a) 以上XRD实验为每个样品测试 3 次, 最大误差 $d \leq 0.02$. 表中所有数据的误差范围为 $\pm 1 \%$

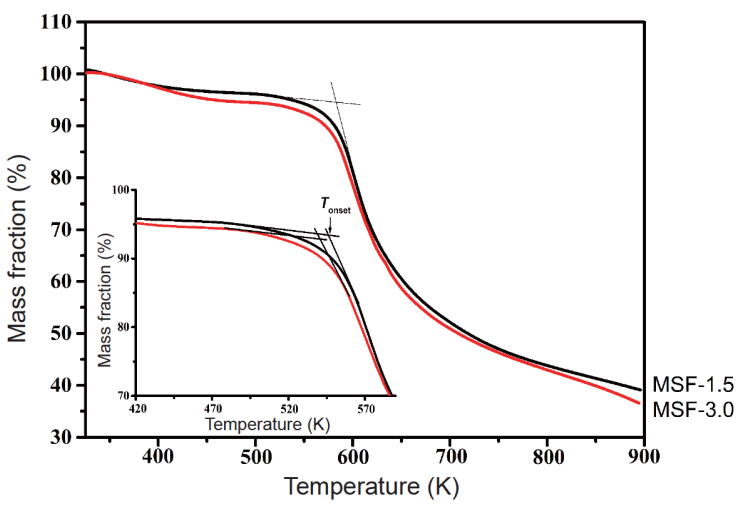

图 4 MSF-1.5 和MSF-3.0的热重曲线图(网络版彩图) Figure 4 TG curves of MSF-1.5 and MSF-3.0 at a heating rate of $10 \mathrm{~K} \mathrm{~min}^{-1}$ (color online).

$(464 \sim 900 \mathrm{~K})$ 的热分解反应进行动力学分析，揭示其老 化时间、热稳定性及反应机理等，为丝素膜在不同应 用领域上的研究提供更多有用信息.

图5是中国家虫丝素膜的不同升温速率 $(2 、 5 、 10$ 和 $15 \mathrm{~K} \mathrm{~min}^{-1}$ )的热重曲线和热重微分曲线. 可以看出, 随着升温速率的增大, 膜的分解曲线向高温方向发生 偏移. 这是由于升温较快，使得材料对温度的热滞后 现象较明显 ${ }^{[38]}$. 由表4中的数据推测, 随着氯化钙浓度 的增大，最大升温速率下其分解温度与最小升温速率 下的差值在逐渐增大. 例如， $1.50 \mathrm{wt} \%$ 浓度制备的中 国丝素膜在 $2 \mathrm{~K} \mathrm{~min}^{-1}$ 时的 $T_{\mathrm{onset}}$ 为 $525.10 \mathrm{~K}$, 在 $15 \mathrm{~K} \mathrm{~min}^{-1}$ 时的 $T_{\text {onset }}$ 为 $551.69 \mathrm{~K}$, 这两者的差值 $\Delta T_{1}$ 为 $26.59 \mathrm{~K}, 3.00 \mathrm{wt} \%$ 浓度制备的膜的 $\Delta T_{1}$ 增加到 $30.32 \mathrm{~K}$. 同时, 泰国丝素膜也出现同样的情况. 这可能是因为材 料中无规线团含量占主导，即无序化程度增加、取向 度降低, 使得其热稳定性降低. 因此, 在相同实验条件 下, 高浓度制备的膜比低浓度下的膜易分解, 其起始分
解温度随着浓度增大而减小, 且受升温速率的影响也 较大.

\subsection{3 丝素膜热分解动力学机理}

以中国家蚕丝素膜为例, 进行详细说明. 从图 $5(\mathrm{~A}$, B)中看出, 约从起始分解温度(Onset温度) $464 \mathrm{~K}$ 开始, 丝素膜的分子骨架断裂，试样开始发生大规模分解反 应，分解速率逐渐增大, MSF-1.5 和MSF-3.0的最大失 重速率所对应的温度不超过 $584 \mathrm{~K}$. 之后, 分解速率开 始逐渐减小. 其中, MSF-1.5试样的DTG主峰中含有一 微小肩峰(约594 774 K). 本文运用TG-DTG技术, 采用 三种方法(Ozawa法、Kissinger法和Vyazovkin法 ${ }^{[39 \sim 41]}$ ) 分别对丝素膜在464 594 K和594 900 K这两个温度区 间的两个分解过程进行了动力学参数(活化能 $E$ 和指前 因子 $\ln A$ )计算, 其过程如下.

将表 5 中数据代入(1)、(2)和(3)式中进行线性回归 拟合, 求得其相应的活化能 $E_{\alpha}$.

$$
\begin{aligned}
& \lg \beta=\lg \left[\frac{A E_{\alpha}}{R g(\alpha)}\right]-2.315-\frac{0.4567 E_{\alpha}}{R T_{\alpha}} \\
& \ln \left(\frac{\beta}{T_{\mathrm{p}}^{2}}\right)=\ln \left(\frac{A R}{E_{\alpha}}\right)-\frac{E}{R T_{\mathrm{p}}} \\
& \ln \left(\frac{\beta}{T_{\alpha}^{2}}\right)=-\frac{E_{\alpha}}{R T_{\alpha}}+\ln \left(\frac{A R}{E_{\alpha} g_{(\alpha)}}\right)
\end{aligned}
$$

其中, $\beta$ 为升温速率 $\left(\mathrm{K} \mathrm{min}^{-1}\right) ; A$ 为指前因子 $\left(\mathrm{s}^{-1}\right) ; E_{\alpha}$ 为 转化率 $\alpha$ 下对应的活化能 $\left(\mathrm{kJ} \mathrm{mol}^{-1}\right) ; g(\alpha)$ 为分解反应的 积分函数; $R$ 为理想气体常数 $\left(8.31 \mathrm{~J} \mathrm{~K}^{-1} \mathrm{~mol}^{-1}\right) ; T_{\mathrm{p}}$ 为最 大失重速率对应的温度 $(\mathrm{K}) ; T_{\alpha}$ 为转化率 $\alpha$ 下对应的温 度 $(\mathrm{K})$.

综合以上拟合结果, 将其计算结果列于表 6 和7. 由 表6中看出，Ozawa法和Vyazovkin法计算MSF-1.5的活 
表 3 两种丝素膜在 $10 \mathrm{~K} \mathrm{~min}^{-1}$ 的升温速率下的测得热分解数据 ${ }^{\mathrm{a}}$

Table 3 Thermal decomposition data of two silk fibroin films measured at a heating rate of $10 \mathrm{~K} \mathrm{~min}^{-1}$ a)

\begin{tabular}{ccccc}
\hline 样品 & 来源 & $T_{\text {onset }}(\mathrm{K})$ & $T_{\mathrm{p}}(\mathrm{K})$ & Residue mass- $T_{\mathrm{p}}(\%)$ \\
\hline MSF-1.5 & 中国家蚕 & 545.81 & 571.86 & 78.46 \\
TSF-1.5 & 泰国家蚕 & 551.03 & 570.53 & 77.80 \\
MSF-3.0 & 中国家虫 & 542.78 & 568.43 & 76.75 \\
TSF-3.0 & 泰国家蚻 & 545.81 & 566.92 & 75.39 \\
\hline
\end{tabular}

a) $T_{\text {onset }}$ 为样品的起始分解温度; $T_{\mathrm{p}}$ 为是TG曲线上分解速率达到最大时所对应的温度, 即DTG曲线上最大峰所对应的峰温

表 4 两种丝素膜的TG热分解特征温度 ${ }^{\mathrm{a})}$

Table 4 The thermal decomposition characteristics of the temperature of two kinds of silk films ${ }^{\text {a) }}$

\begin{tabular}{|c|c|c|c|c|c|c|c|c|c|}
\hline \multirow{2}{*}{ 样品 } & \multicolumn{4}{|c|}{ 不同升温速率的 $T_{\text {onset }}(\mathrm{K})$} & \multicolumn{4}{|c|}{ 不同升温速率的 $T_{\mathrm{p}}(\mathrm{K})$} & \multirow{2}{*}{$\Delta T_{1}(\mathrm{~K})$} \\
\hline & $2 \mathrm{~K} \mathrm{~min}^{-1}$ & $5 \mathrm{~K} \mathrm{~min}^{-1}$ & $10 \mathrm{~K} \mathrm{~min}^{-1}$ & $15 \mathrm{~K} \mathrm{~min}^{-1}$ & $2 \mathrm{~K} \mathrm{~min}^{-1}$ & $5 \mathrm{~K} \mathrm{~min}^{-1}$ & $10 \mathrm{~K} \mathrm{~min}^{-1}$ & $15 \mathrm{~K} \mathrm{~min}^{-1}$ & \\
\hline MSF-1.5 & 525.10 & 538.00 & 545.81 & 551.69 & 551.27 & 565.58 & 571.86 & 581.49 & 26.59 \\
\hline MSF-3.0 & 519.08 & 532.85 & 542.78 & 549.40 & 550.97 & 562.35 & 568.43 & 580.00 & 30.32 \\
\hline TSF-1.5 & 531.33 & 540.45 & 551.03 & 557.68 & 546.54 & 559.09 & 570.53 & 578.10 & 26.35 \\
\hline TSF-3.0 & 522.79 & 538.48 & 548.27 & 550.11 & 541.63 & 554.84 & 566.92 & 573.02 & 28.32 \\
\hline
\end{tabular}

a) $\Delta T_{1}$ 为同一丝素在最大升温速率与最小升温速率下的起始分解温度差; $T_{\mathrm{p}}$ 为是 TG曲线上分解速率达到最大时所对应的温度, 即 DTG 曲线上最大峰所对应的峰温
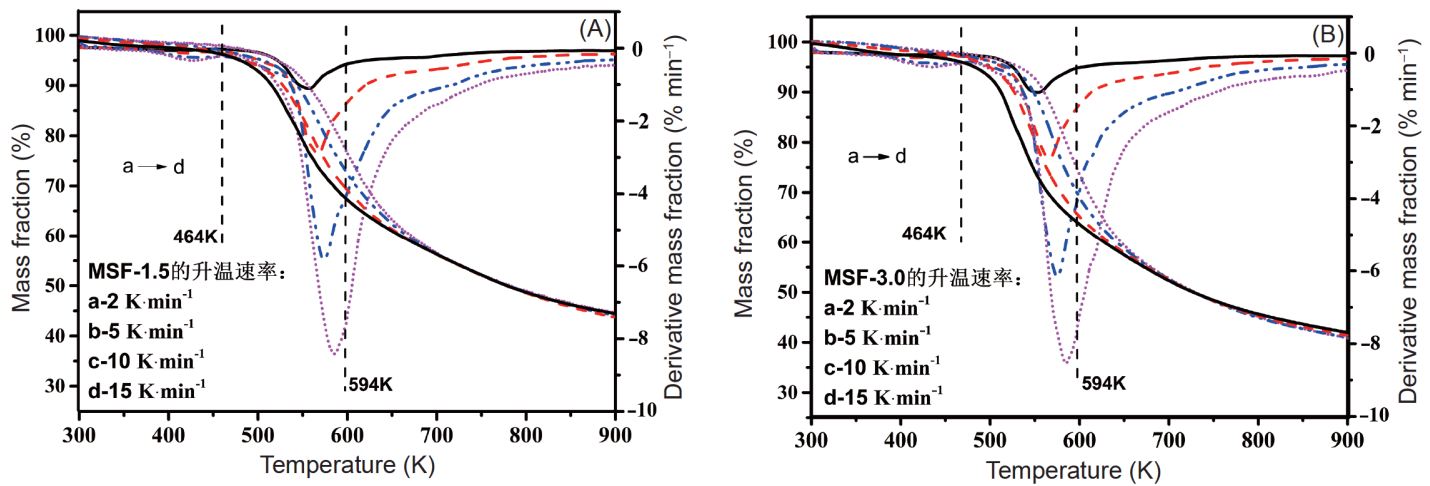

图 5 MSF-1.5 (A)和MSF-3.0 (B)在不同升温速率下的TG和DTG曲线. (a) $2 \mathrm{~K} \mathrm{~min}^{-1}$; (b) $5 \mathrm{~K} \mathrm{~min}^{-1}$; (c) $10 \mathrm{~K} \mathrm{~min}{ }^{-1}$; (d) $15 \mathrm{~K} \mathrm{~min}^{-1}$ (网络版彩图)

Figure 5 TG and DTG curves of (A) MSF-1.5 and (B) MSF-3.0 at different heating rates. (a) $2 \mathrm{~K} \mathrm{~min}^{-1}$; (b) $5 \mathrm{~K} \mathrm{~min}^{-1}$; (c) $10 \mathrm{~K} \mathrm{~min}{ }^{-1}$; (d) $15 \mathrm{~K} \mathrm{~min}^{-1}$ (color online).

化能分别为 158.35 和 $157.47 \mathrm{~kJ} \mathrm{~mol}^{-1}$, MSF-3.0的活化 能分别154.92和 $154.08 \mathrm{~kJ} \mathrm{~mol}^{-1}$ ，这两种方法计算的活 化能数值很接近, Kissinger法计算MSF-1.5 和MSF-3.0 的活化能分别是 157.95 和 $153.98 \mathrm{~kJ} \mathrm{~mol}^{-1}$; 同样, 用 Ozawa法和Vyazovkin法计算TSF-1.5 的活化能分别为 189.81 和 $190.31 \mathrm{~kJ} \mathrm{~mol}^{-1}$, TSF-3.0的活化能分别 174.88 和 $173.85 \mathrm{~kJ} \mathrm{~mol}^{-1}$. 用Kissinger法计算TSF-1.5 和TSF-
3.0 的活化能分别是 189.55 和 $174.84 \mathrm{~kJ} \mathrm{~mol}^{-1}$. 从数值 上可以发现，Ozawa法、Vyazovkin法和Kissinger法的 计算结果均非常接近. 说明通过这三种方法所得到的 两种丝素膜的热分解活化能较为准确可信. 为了得到 更加准确的活化能，我们分别将两种丝素膜下三种方 法所得的活化能取平均值, 即MSF-1.5 的平均活化能 $E$ 为 $157.92 \mathrm{~kJ} \mathrm{~mol}^{-1}$ ，指前因子 $\ln A$ 为 $25.57 \mathrm{~s}^{-1}, M S F-3.0$ 
表 5 不同升温速率下中国丝素膜的热分解温度

Table 5 Thermal decomposition temperatures of MSF-1.5 and MSF-3.0 at different heating rates

\begin{tabular}{|c|c|c|c|c|c|c|c|c|c|}
\hline & \multicolumn{4}{|c|}{$\beta\left(\mathrm{K} \mathrm{min}^{-1}\right)(464 \sim 594 \mathrm{~K})^{\mathrm{a})}$} & \multicolumn{4}{|c|}{$\beta\left(\mathrm{K} \mathrm{min}^{-1}\right)(594 \sim 900 \mathrm{~K})^{\mathrm{a})}$} & \multirow{2}{*}{$\alpha^{\mathrm{b})}$} \\
\hline & $2 \mathrm{~K}$ & $5 \mathrm{~K}$ & $10 \mathrm{~K}$ & $15 \mathrm{~K}$ & $2 \mathrm{~K}$ & $5 \mathrm{~K}$ & $10 \mathrm{~K}$ & $15 \mathrm{~K}$ & \\
\hline \multirow{17}{*}{$\begin{array}{c}T_{a}(\mathrm{~K})^{\mathrm{c})} \\
(\mathrm{MSF}-1.5)\end{array}$} & 489.54 & 497.81 & 507.45 & 518.30 & 594.90 & 597.28 & 608.06 & 619.68 & 0.10 \\
\hline & 501.06 & 509.41 & 518.89 & 531.07 & 596.38 & 601.39 & 611.75 & 623.41 & 0.15 \\
\hline & 512.58 & 521.01 & 530.32 & 543.83 & 599.36 & 605.57 & 615.53 & 627.14 & 0.20 \\
\hline & 519.44 & 526.47 & 540.27 & 547.72 & 599.25 & 610.97 & 620.51 & 631.71 & 0.25 \\
\hline & 526.30 & 531.93 & 550.22 & 551.61 & 594.98 & 616.36 & 625.48 & 636.27 & 0.30 \\
\hline & 529.29 & 536.68 & 549.91 & 554.96 & 597.62 & 623.46 & 630.88 & 641.25 & 0.35 \\
\hline & 532.27 & 541.43 & 549.59 & 558.31 & 598.25 & 629.63 & 636.27 & 646.22 & 0.40 \\
\hline & 534.49 & 543.38 & 553.72 & 561.02 & 607.38 & 638.76 & 644.57 & 652.86 & 0.45 \\
\hline & 536.70 & 545.33 & 557.85 & 563.73 & 616.50 & 647.88 & 652.86 & 659.56 & 0.50 \\
\hline & 236.75 & 547.53 & 559.93 & 567.04 & 627.21 & 658.59 & 662.78 & 668.11 & 0.55 \\
\hline & 536.70 & 549.73 & 562.01 & 570.34 & 637.91 & 669.29 & 672.69 & 676.71 & 0.60 \\
\hline & 538.44 & 551.53 & 563.72 & 571.02 & 661.22 & 692.69 & 695.98 & 700.19 & 0.65 \\
\hline & 540.17 & 553.33 & 565.43 & 571.70 & 684.52 & 715.93 & 719.27 & 723.66 & 0.70 \\
\hline & 543.51 & 554.92 & 566.98 & 573.43 & 702.47 & 733.85 & 750.97 & 740.14 & 0.75 \\
\hline & 546.84 & 556.51 & 568.52 & 575.16 & 720.41 & 751.79 & 752.67 & 756.62 & 0.80 \\
\hline & 547.91 & 557.99 & 569.97 & 576.90 & 634.70 & 666.08 & 671.76 & 679.71 & 0.85 \\
\hline & 548.98 & 559.47 & 571.41 & 578.64 & 548.98 & 598.36 & 590.85 & 602.79 & 0.90 \\
\hline$T_{\mathrm{p}}(\mathrm{K})^{\mathrm{d})}$ & 546.54 & 559.09 & 570.53 & 578.10 & 580.23 & 597.46 & 613.83 & 619.63 & \\
\hline \multirow{17}{*}{$\begin{array}{c}T_{a}(\mathrm{~K})^{\mathrm{c})} \\
(\mathrm{MSF}-3.0)\end{array}$} & 488.19 & 497.76 & 507.39 & 517.65 & 594.14 & 604.84 & 614.28 & 623.11 & 0.10 \\
\hline & 499.73 & 508.59 & 518.58 & 529.61 & 597.92 & 608.94 & 617.43 & 626.25 & 0.15 \\
\hline & 511.26 & 519.42 & 529.76 & 541.56 & 601.69 & 613.03 & 620.58 & 629.39 & 0.20 \\
\hline & 517.97 & 525.26 & 538.16 & 547.25 & 606.73 & 618.07 & 624.99 & 633.49 & 0.25 \\
\hline & 524.68 & 531.09 & 546.56 & 552.93 & 611.77 & 623.14 & 629.39 & 637.58 & 0.30 \\
\hline & 527.96 & 536.30 & 548.19 & 555.84 & 619.45 & 631.89 & 634.87 & 642.11 & 0.35 \\
\hline & 531.24 & 541.50 & 549.81 & 558.74 & 627.12 & 634.67 & 640.34 & 646.63 & 0.40 \\
\hline & 533.23 & 544.36 & 553.47 & 561.58 & 636.25 & 642.86 & 646.95 & 651.67 & 0.45 \\
\hline & 535.21 & 547.21 & 557.12 & 564.41 & 645.37 & 651.04 & 653.56 & 656.71 & 0.50 \\
\hline & 536.26 & 548.55 & 558.94 & 566.28 & 656.72 & 660.18 & 662.28 & 663.85 & 0.55 \\
\hline & 537.31 & 549.88 & 560.76 & 568.14 & 668.07 & 669.32 & 670.99 & 670.99 & 0.60 \\
\hline & 538.58 & 551.88 & 562.33 & 569.28 & 677.67 & 675.63 & 683.66 & 684.36 & 0.65 \\
\hline & 539.84 & 553.88 & 563.90 & 571.42 & 689.64 & 690.06 & 693.47 & 695.06 & 0.70 \\
\hline & 541.89 & 555.33 & 565.38 & 572.93 & 699.28 & 695.67 & 796.61 & 709.03 & 0.75 \\
\hline & 543.93 & 556.78 & 566.86 & 574.43 & 715.06 & 716.31 & 722.15 & 722.99 & 0.80 \\
\hline & 544.31 & 558.17 & 568.17 & 575.86 & 731.31 & 732.36 & 742.25 & 740.93 & 0.85 \\
\hline & 544.68 & 559.56 & 569.48 & 577.28 & 747.56 & 748.43 & 756.2 & 758.81 & 0.90 \\
\hline$T_{\mathrm{p}}(\mathrm{K})^{\mathrm{d})}$ & 541.63 & 554.84 & 566.92 & 573.02 & 558.34 & 581.66 & 597.77 & 615.71 & \\
\hline
\end{tabular}

a) $\beta$ 是升温速率; b) $\alpha$ 表示转化率; c) $T_{\alpha}$ 为不同转化率下对应温度; d) $T_{\mathrm{p}}$ 是TG曲线上分解速率达到最大时所对应的温度, 即DTG曲线上最 大峰所对应的峰温 
表 6 不同转化率下中国丝素膜的动力学参数 ${ }^{a}$

Table 6 Kinetic parameters of MSF-1.5 and MSF-3.0 at different conversions ${ }^{\text {a) }}$

\begin{tabular}{|c|c|c|c|c|c|c|c|c|c|c|c|c|}
\hline \multirow{3}{*}{$\alpha_{1}^{\mathrm{b})}$} & \multicolumn{4}{|c|}{ Ozawa法 } & \multicolumn{4}{|c|}{ Kissinger法 } & \multicolumn{4}{|c|}{ Vyazovkin法 } \\
\hline & \multicolumn{2}{|c|}{ MSF-1.5 } & \multicolumn{2}{|c|}{ MSF-3.0 } & \multicolumn{2}{|c|}{ MSF-1.5 } & \multicolumn{2}{|c|}{ MSF-3.0 } & \multicolumn{2}{|c|}{ MSF-1.5 } & \multicolumn{2}{|c|}{ MSF-3.0 } \\
\hline & $\begin{array}{c}E_{\alpha} \\
\left(\mathrm{kJ} \mathrm{mol}^{-1}\right)^{\mathrm{c})}\end{array}$ & $r$ & $\begin{array}{c}E_{\alpha} \\
\left(\mathrm{kJ} \mathrm{mol}^{-1}\right)^{\mathrm{c})}\end{array}$ & $r$ & $\begin{array}{c}E_{\alpha} \\
\left(\mathrm{kJ} \mathrm{mol}^{-1}\right)^{\mathrm{c})}\end{array}$ & $r$ & $\begin{array}{c}E_{\alpha} \\
\left(\mathrm{kJ} \mathrm{mol}^{-1}\right)^{\mathrm{c})}\end{array}$ & $r$ & $\begin{array}{c}E_{\alpha} \\
\left(\mathrm{kJ} \mathrm{mol}^{-1}\right)^{\mathrm{c})}\end{array}$ & $r$ & $\begin{array}{c}E_{\alpha} \\
\left(\mathrm{kJ} \mathrm{mol}^{-1}\right)^{\mathrm{c})}\end{array}$ & $r$ \\
\hline 0.10 & 139.00 & 0.978 & 136.83 & 0.986 & - & - & - & - & 137.82 & 0.975 & 135.55 & 0.984 \\
\hline 0.15 & 139.53 & 0.969 & 140.28 & 0.978 & - & - & - & - & 137.99 & 0.976 & 137.89 & 0.980 \\
\hline 0.20 & 139.82 & 0.966 & 142.90 & 0.973 & - & - & - & - & 138.27 & 0.961 & 141.54 & 0.970 \\
\hline 0.25 & 145.29 & 0.957 & 145.45 & 0.970 & - & - & - & - & 145.28 & 0.987 & 145.79 & 0.998 \\
\hline 0.30 & 150.54 & 0.958 & 149.32 & 0.974 & - & - & - & - & 149.36 & 0.953 & 148.09 & 0.971 \\
\hline 0.35 & 168.45 & 0.991 & 150.25 & 0.980 & - & - & - & - & 168.57 & 0.968 & 167.98 & 0.987 \\
\hline 0.40 & 183.41 & 0.990 & 174.35 & 0.992 & - & - & - & - & 183.83 & 0.989 & 174.30 & 0.991 \\
\hline 0.45 & 180.25 & 0.990 & 170.25 & 0.992 & - & - & - & - & 178.92 & 0.996 & 170.26 & 0.992 \\
\hline 0.50 & 171.00 & 0.991 & 165.51 & 0.999 & - & - & - & - & 170.69 & 0.990 & 164.93 & 0.999 \\
\hline 0.55 & 164.32 & 0.997 & 160.29 & 0.995 & - & - & - & - & 158.39 & 0.995 & 160.29 & 0.993 \\
\hline 0.60 & 144.54 & 0.998 & 146.97 & 0.999 & - & - & - & - & 142.69 & 0.998 & 156.69 & 0.999 \\
\hline 0.65 & 154.37 & 0.996 & 150.40 & 0.997 & - & - & - & - & 150.48 & 0.994 & 155.01 & 0.995 \\
\hline 0.70 & 153.90 & 0.999 & 156.73 & 0.999 & - & - & - & - & 152.62 & 0.999 & 155.61 & 0.999 \\
\hline 0.75 & 167.29 & 0.996 & 158.49 & 0.999 & - & - & - & - & 162.39 & 0.993 & 159.28 & 0.999 \\
\hline 0.80 & 172.62 & 0.994 & 162.82 & 0.999 & - & - & - & - & 172.22 & 0.994 & 161.95 & 0.999 \\
\hline 0.85 & 170.27 & 0.995 & 160.37 & 0.999 & - & - & - & - & 170.48 & 0.992 & 158.38 & 0.999 \\
\hline 0.90 & 167.62 & 0.996 & 155.07 & 0.999 & - & - & - & - & 167.23 & 0.995 & 153.79 & 0.999 \\
\hline $\begin{array}{c}\overline{E_{\alpha}} \\
\left(\mathrm{kJ} \mathrm{mol}^{-1}\right)^{\mathrm{d})}\end{array}$ & 158.35 & - & 154.92 & - & 157.95 & 0.999 & 153.98 & 0.999 & 157.47 & - & 154.08 & - \\
\hline $\ln A\left(s^{-1}\right)^{\mathrm{e})}$ & - & - & - & - & 25.57 & & 25.38 & & - & - & - & - \\
\hline
\end{tabular}

a) 动力学参数是在 $15 \mathrm{~K}$ 升温速率下从 $\mathrm{TG}$ 曲线上 $464 \sim 594 \mathrm{~K}$ 获得; b) $\alpha_{1}$ 是不同转化率; c) $E_{\alpha}$ 是降解过程中的活化能; d) $\overline{E_{\alpha}}$ 是利用某一种方 程计算的平均活化能; e) $\ln A$ 是指前因子的自然对数

的平均活化能 $\bar{E}$ 为 $154.89 \mathrm{~kJ} \mathrm{~mol}^{-1}$, 指前因子 $\ln A$ 为 $25.38 \mathrm{~s}^{-1}$; TSF-1.5 的平均活化能 $E$ 为 $189.89 \mathrm{~kJ} \mathrm{~mol}^{-1}$, 指前因子 $\ln A$ 为 $39.47 \mathrm{~s}^{-1}, \mathrm{TSF}-3.0$ 的平均活化能 $\bar{E}$ 为 $174.52 \mathrm{~kJ} \mathrm{~mol}^{-1}$, 指前因子 $\ln A$ 为 $36.38 \mathrm{~s}^{-1}$. 根据膜材料 的动力学计算结果来看, 高浓度氯化钲下制备的丝素

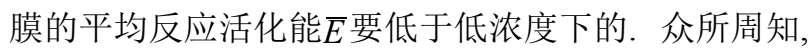
反应活化能越低，表明反应活性就越强，分解反应就 越容易发生，因此，高浓度氯化钻下制备的丝素膜就 越易发生分解, 这与丝素膜TG分析的结论相一致.

另外, 从表7可以发现, 用Ozawa法和Vyazovkin法 这两种方法计算MSF-1.5 和MSF-3.0在594 900 K区间 的活化能与Kissinger法计算的活化能差异较大，且这 两种方法计算的活化能随着转化率的增大均呈现先增
大后减小的变化趋势，变化较大. 本文着重对464 $594 \mathrm{~K}$ 范围内的分解过程进行热分析动力学研究.

\subsection{4 丝素蛋白膜热力学参数测定}

通过将表6中计算的活化能、指前因子代入到式 (4 6), 计算其DTG曲线上的峰温 $T_{\mathrm{p}}$ 的热力学参数: 活 化焓、熵以及吉布斯自由能, 并将结果列于表 8 .

$$
\begin{aligned}
& -\frac{E}{R T}+\ln A=-\frac{\Delta G^{\ddagger}}{R T}+\ln \frac{k_{\mathrm{B}} T}{h} \\
& \Delta H^{\neq}=E-R T \\
& \Delta G^{\neq}=\Delta H^{\neq}-T \Delta S^{\ddagger}
\end{aligned}
$$

其中, $\Delta G^{\neq}$为活化吉布斯自由能, $\Delta H^{\neq}$为活化焓, $\Delta S^{\neq}$为 
表 7 不同转化率下中国丝素膜的动力学参数 ${ }^{a}$

Table 7 Kinetic parameters of MSF-1.5 and MSF-3.0 at different conversions ${ }^{\text {a) }}$

\begin{tabular}{|c|c|c|c|c|c|c|c|c|c|c|c|c|}
\hline \multirow{3}{*}{$\alpha_{2}^{\mathrm{b})}$} & \multicolumn{4}{|c|}{ Ozawa法 } & \multicolumn{4}{|c|}{ Kissinger法 } & \multicolumn{4}{|c|}{ Vyazovkin法 } \\
\hline & \multicolumn{2}{|c|}{ MSF-1.5 } & \multicolumn{2}{|c|}{ MSF-3.0 } & \multicolumn{2}{|c|}{ MSF-1.5 } & \multicolumn{2}{|c|}{ MSF-3.0 } & \multicolumn{2}{|c|}{ MSF-1.5 } & \multicolumn{2}{|c|}{ MSF-3.0 } \\
\hline & $\begin{array}{c}E_{\alpha} \\
\left(\mathrm{kJ} \mathrm{mol}^{-1}\right)^{\mathrm{c})}\end{array}$ & $r$ & $\begin{array}{c}E_{\alpha} \\
\left(\mathrm{kJ} \mathrm{mol}^{-1}\right)^{\mathrm{c})}\end{array}$ & $r$ & $\begin{array}{c}E_{\alpha} \\
\left(\mathrm{kJ} \mathrm{mol}^{-1}\right)^{\mathrm{c})}\end{array}$ & $r$ & $\begin{array}{c}E_{\alpha} \\
\left(\mathrm{kJ} \mathrm{mol}^{-1}\right)^{\mathrm{c})}\end{array}$ & $r$ & $\begin{array}{c}E_{\alpha} \\
\left(\mathrm{kJ} \mathrm{mol}^{-1}\right)^{\mathrm{c})}\end{array}$ & $r$ & $\begin{array}{c}E_{\alpha} \\
\left(\mathrm{kJ} \mathrm{mol}^{-1}\right)^{\mathrm{c})}\end{array}$ & $r$ \\
\hline 0.10 & 174.76 & 0.996 & 181.59 & 0.994 & - & - & - & - & 179.65 & 0.996 & 186.96 & 0.993 \\
\hline 0.15 & 177.11 & 0.997 & 192.12 & 0.995 & - & - & - & - & 187.88 & 0.997 & 199.7 & 0.992 \\
\hline 0.20 & 192.87 & 0.996 & 197.33 & 0.994 & - & - & - & - & 198.58 & 0.996 & 203.37 & 0.993 \\
\hline 0.25 & 207.84 & 0.998 & 207.36 & 0.993 & - & - & - & - & 202.45 & 0.995 & 211.69 & 0.992 \\
\hline 0.30 & 224.56 & 0.996 & 219.85 & 0.993 & - & - & - & - & 231.17 & 0.996 & 226.92 & 0.993 \\
\hline 0.35 & 247.01 & 0.999 & 275.39 & 0.991 & - & - & - & - & 253.07 & 0.993 & 289.72 & 0.991 \\
\hline 0.40 & 275.56 & 0.990 & 301.31 & 0.992 & - & - & - & - & 285.25 & 0.990 & 312.38 & 0.992 \\
\hline 0.45 & 297.61 & 0.992 & 266.72 & 0.999 & - & - & - & - & 355.46 & 0.997 & 292.72 & 0.991 \\
\hline 0.50 & 411.03 & 0.993 & 549.35 & 0.994 & - & - & - & - & 427.39 & 0.994 & 572.98 & 0.993 \\
\hline 0.55 & 648.11 & 0.992 & 896.33 & 0.993 & - & - & - & - & 521.53 & 0.995 & 601.8 & 0.994 \\
\hline 0.60 & 743.69 & 0.991 & 1073.93 & 0.930 & - & - & - & - & 776.91 & 0.992 & 1123.95 & 0.929 \\
\hline 0.65 & 597.09 & 0.947 & 564.08 & 0.978 & - & - & - & - & 602.53 & 0.987 & 800.5 & 0.994 \\
\hline 0.70 & 502.05 & 0.658 & 770.73 & 0.946 & - & - & - & - & 522.02 & 0.650 & 804.65 & 0.944 \\
\hline 0.75 & 786.09 & 0.789 & 647.12 & 0.987 & - & - & - & - & 702.55 & 0.943 & 702.52 & 0.956 \\
\hline 0.80 & 1215.65 & 0.949 & 597.06 & 0.930 & - & - & - & - & 1271.85 & 0.949 & 621.45 & 0.928 \\
\hline 0.85 & 669.92 & 0.994 & 297.17 & 0.976 & - & - & - & - & 402.55 & 0.957 & 202.42 & 0.937 \\
\hline 0.90 & 164.75 & 0.995 & 151.92 & 0.999 & - & - & - & - & 169.41 & 0.995 & 156.03 & 0.999 \\
\hline $\begin{array}{c}\overline{E_{\alpha}}(\mathrm{kJ} \\
\left.\mathrm{mol}^{-1}\right)^{\mathrm{d})}\end{array}$ & 503.47 & - & 486.15 & - & 134.46 & 0.998 & 95.94 & 0.994 & 453.46 & - & 477.36 & - \\
\hline $\ln A\left(s^{-1}\right)^{\mathrm{e})}$ & - & - & - & - & 18.35 & & 12.18 & & - & - & - & - \\
\hline
\end{tabular}

a) 动力学参数是在 $15 \mathrm{~K}$ 升温速率下从 $\mathrm{TG}$ 曲线上 $594 \sim 900 \mathrm{~K}$ 获得; b) $\alpha_{1}$ 是不同转化率; c) $E_{\alpha}$ 是降解过程中的活化能; d) $\overline{E_{\alpha}}$ 是利用某一种方 程计算的平均活化能; e) $\ln A$ 是指前因子的自然对数

活化熵, $h$ 为普朗克常数 $\left(6.63 \times 10^{-34} \mathrm{~J} \mathrm{~s}^{-1}\right), R$ 为气体常 数 $\left(8.31 \mathrm{~J} \mathrm{~mol}^{-1} \mathrm{~K}^{-1}\right), k_{\mathrm{B}}$ 为玻尔兹曼常数 $(1.38 \times$ $\left.10^{-23} \mathrm{~J} \mathrm{~K}^{-1}\right), T$ 为绝对温度 $(\mathrm{K})$.

从表 8 中看出, $\Delta G^{\ddagger>0}$ 表明丝素膜的分解过程并不 是自发进行的, 需要外界提供能量才会发生热分解. 表 8 中MSF-1.5 和MSF-3.0这些热力学参数都为正值, 说 明TSF-1.5和TSF-3.0的分解过程需要吸收一定的能量 $\left(\Delta G^{\ngtr>0}\right)$, 在丝素膜分解过程中非共价键的断裂 $\left(\Delta H^{\ddagger>0}\right)$ 及丝素蛋白的结构打开增加了体系的无序性 $\left(\Delta S^{\ddagger>0}\right)^{[42]}$. 但我们发现MSF-3.0不同的升温速率所对

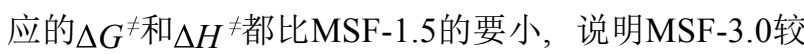
易发生分解行为, MSF-3.0中的非共价键数量较MSF1.5 要少, 即较高浓度的钻离子对丝素蛋白分子链段间
的非共价键(氢键)破坏程度较大, 使得其链段无序程 度增大, 即MSF-3.0较MSF-1.5的结晶程度要低, 这一 结果与FTIR和XRD分析结果一致. 此外, 由于反应速 率受活化能的影响：活化能越大，分解反应速率越 慢 ${ }^{[43]}$. 从表 8 中看出MSF-1.5 的平均活化能 $\bar{E}$ 比MSF3.0 大, 所以MSF-1.5的热分解反应速率较MSF-3.0要缓 慢, MSF-1.5样品的热稳定性比MSF-3.0要好.

\subsection{5 丝素膜的等温失重时间比较}

$\log t \cong \log \left(\frac{E}{\beta R}\right)-2.315+\frac{E}{2.303 R T_{\mathrm{H}}}-0.4567 \frac{E}{R T}$

利用式(7)可以计算丝素膜的等温失重时间 ${ }^{[4]}$, 其 中, $\beta$ (升温速率)为 $15 \mathrm{~K} \mathrm{~min}^{-1}, T$ 在 $\beta$ 升温速率下达到某 
表 8 两种丝素膜在 $T_{\mathrm{p}}$ 温度下的热力学参数 ${ }^{\mathrm{a})}$

Table 8 Thermodynamic data of two kinds of silk fibroin films at $T_{\mathrm{p}}^{\text {a) }}$

\begin{tabular}{|c|c|c|c|c|c|c|c|}
\hline 样品 & 来源 & 氯化钲浓度 (\%) & $\beta\left(\mathrm{K} \mathrm{min}^{-1}\right)$ & $T_{\mathrm{p}}(\mathrm{K})^{\mathrm{b})}$ & $\Delta G^{\neq}\left(\mathrm{kJ} \mathrm{mol}^{-1}\right)^{\mathrm{c})}$ & $\Delta H^{\neq}\left(\mathrm{kJ} \mathrm{mol}^{-1}\right)^{\mathrm{d})}$ & $\left.\Delta S^{\neq}\left(\mathrm{J} \mathrm{mol}^{-1} \mathrm{~K}^{-1}\right)^{\mathrm{e}}\right)$ \\
\hline \multirow{4}{*}{ MSF-1.5 } & \multirow{4}{*}{ 中国家蚕 } & \multirow{4}{*}{$1.5 \%$} & 2 & 546.44 & 148.67 & 190.35 & 77.36 \\
\hline & & & 5 & 559.09 & 147.56 & 189.79 & 77.45 \\
\hline & & & 10 & 570.53 & 146.36 & 190.34 & 76.23 \\
\hline & & & 15 & 578.10 & 145.47 & 188.35 & 76.15 \\
\hline \multirow{4}{*}{ MSF-3.0 } & \multirow{4}{*}{ 中国家蚕 } & \multirow{4}{*}{$3.0 \%$} & 2 & 541.63 & 147.35 & 176.01 & 51.35 \\
\hline & & & 5 & 554.84 & 147.25 & 175.04 & 51.04 \\
\hline & & & 10 & 566.92 & 146.02 & 173.56 & 50.86 \\
\hline & & & 15 & 573.02 & 145.27 & 172.97 & 49.79 \\
\hline \multirow{4}{*}{ TSF-1.5 } & \multirow{4}{*}{ 泰国家蚕 } & \multirow{4}{*}{$1.5 \%$} & 2 & 552.65 & 146.46 & 185.69 & 70.59 \\
\hline & & & 5 & 563.64 & 145.57 & 185.46 & 70.38 \\
\hline & & & 10 & 572.29 & 145.07 & 185.67 & 70.25 \\
\hline & & & 15 & 579.90 & 145.48 & 189.79 & 70.36 \\
\hline \multirow{4}{*}{ TSF-3.0 } & \multirow{4}{*}{ 泰国家蚕 } & \multirow{4}{*}{$3.0 \%$} & 2 & 549.25 & 145.04 & 169.58 & 44.59 \\
\hline & & & 5 & 562.34 & 145.38 & 169.43 & 43.86 \\
\hline & & & 10 & 571.78 & 144.02 & 169.59 & 43.69 \\
\hline & & & 15 & 578.22 & 144.57 & 169.04 & 43.27 \\
\hline
\end{tabular}

a) 热力学参数是从 $\mathrm{TG}$ 曲线上 $464 \sim 594 \mathrm{~K}$ 获得, 其中升温速率为 $2 、 5 、 10$ 和 $15 \mathrm{~K} \mathrm{~min}^{-1}$; b) $T_{\mathrm{p}}$ 为 $\mathrm{TG}$ 曲线上分解速率达到最大时所对应的温 度, 即DTG曲线上最大峰所对应的峰温; c) $\Delta G^{\neq}$是吉布斯自由能; d) $\Delta H^{\neq}$是活化能; e) $\Delta S^{\neq}$是活化熵

失重量时所对应的温度. 通过式(7)可计算任意温度 $T_{\mathrm{H}}$ 下达到某一失重量所需的时间 $t$. 经计算, MSF-3.0在 $464 \mathrm{~K}$ 下失重 $0.50 \%$ 所需时间为 $5.2 \mathrm{~s}$ 左右，而MSF-1.5 在 $464 \mathrm{~K}$ 下失重 $0.50 \%$ 所需时间则约为 $13.5 \mathrm{~s}$. 说明 MSF-1.5较MSF-3.0有更很好的热稳定性. 在之前的研 究中 ${ }^{[26]}$, 我们选用泰国家蚕丝(TSF)在相同氯化钙浓度 体系下制备了丝素膜, 并进行了等温时间的比较, 其结 果列于表 9. TSF-3.0在 $464 \mathrm{~K}$ 下失重 $0.50 \%$ 所需时间为 $5.5 \mathrm{~s}$ 左右，而TSF-1.5在 $464.15 \mathrm{~K}$ 下失重 $0.50 \%$ 所需时 间则约为 $52.6 \mathrm{~s}$, 说明在相同浓度体系下制备得到的丝 素膜, TSF具有更好的热稳定性.

\subsection{6 丝素膜的热分解最可几机理函数判定}

本研究中采用Achar和Coats-Redfern两种方法对 MSF-1.5进行热分解机理推测, Achar法和Coats-Redfern法分别如下 ${ }^{[45]}$ :

$$
\begin{gathered}
\ln \frac{\mathrm{d} \alpha / \mathrm{d} t}{f(\alpha)}=\ln A-\frac{E_{\alpha}}{R T_{\alpha}} \\
\ln \frac{g(\alpha)}{T_{\alpha}^{2}}=\ln \frac{A R}{\beta E_{\alpha}}-\frac{E_{\alpha}}{R T_{\alpha}}
\end{gathered}
$$

表 9 两种丝素膜在 $464 \mathrm{~K}$ 下失重 $0.50 \%$ 所需的时间

\begin{tabular}{|c|c|c|c|}
\hline 样品 & 来源 & 氯化钲浓度 (\%) & $t(\mathrm{~s})$ \\
\hline MSF-1.5 & 中国家蚕 & 1.5 & 13.8 \\
\hline TSF-1.5 & 泰国家蚌 & 1.5 & 52.6 \\
\hline MSF-3.0 & 中国家虫 & 3.0 & 5.4 \\
\hline TSF-3.0 & 泰国家虫 & 3.0 & 5.5 \\
\hline
\end{tabular}

Table 9 The time of weightlessness for $0.50 \%$ under $464 \mathrm{~K}$

其中, $\alpha$ 为转化率; $f(\alpha)$ 为微分形式机理函数; $g(\alpha)$ 为积 分形式机理函数; $\beta$ 为升温速率 $\left(\mathrm{K} \mathrm{mol}^{-1}\right) ; \ln A$ 为指前因 子 $\left(\mathrm{s}^{-1}\right) ; E_{\alpha}$ 为转化率 $\alpha$ 下对应的活化能 $\left(\mathrm{kJ} \mathrm{mol}^{-1}\right) ; R$ 为 理想气体常数 $\left(8.31 \mathrm{~J} \mathrm{~K}^{-1} \mathrm{~mol}^{-1}\right) ; T_{\alpha}$ 为转化率 $\alpha$ 下对应 的温度 $(K)$.

将 TG和DTG曲线(图5)中 $15 \mathrm{~K} \mathrm{~min}^{-1}$ 升温速率下 MSF-1.5和MSF-3.0的不同转化率所对应动力学基础 数据列于表 10 . 将表 10 中的数据分别代入式(8)和(9) 中，再进行线性拟合，求出不同机理函数所对应的动 力学参数, 如活化能 $E_{\alpha}$ 、指前因子 $\ln A$ 和相关系数 $r$ (表 11和12).

根据文献 ${ }^{[46]}:$ (1) $E_{\alpha}$ 和 $\ln A$ 符合热分解反应的一般 
刘其春等: 比较两种丝素膜的结构、热分解机理与热力学参数

表 10 中国丝素膜的热分解动力学基础数据 ${ }^{a}$

Table 10 Kinetics of thermal decomposition based data of China silk film ${ }^{\text {a) }}$

\begin{tabular}{|c|c|c|c|c|c|c|c|c|c|}
\hline \multirow{2}{*}{ 编号 } & \multirow{2}{*}{$\alpha^{\mathrm{b})}$} & \multicolumn{4}{|c|}{$T_{\alpha}(\mathrm{K})^{\mathrm{c})}$} & \multicolumn{4}{|c|}{$\left.(\mathrm{d} \alpha / \mathrm{d} t)\left(\% \min ^{-1}\right)^{\mathrm{d}}\right)$} \\
\hline & & MSF-1.5 & TSF-1.5 & MSF-3.0 & TSF-3.0 & MSF-1.5 & TSF-1.5 & MSF-3.0 & TSF-3.0 \\
\hline 1 & 0.10 & 524.30 & 544.19 & 517.65 & 527.12 & 8.90 & 10.79 & 4.37 & 5.42 \\
\hline 2 & 0.15 & 534.07 & 549.96 & 529.6 & 535.99 & 12.84 & 8.24 & 8.59 & 8.10 \\
\hline 3 & 0.20 & 543.83 & 555.73 & 541.56 & 544.87 & 16.77 & 10.45 & 12.81 & 10.78 \\
\hline 4 & 0.25 & 547.72 & 560.37 & 547.25 & 549.09 & 18.62 & 13.29 & 18.46 & 14.60 \\
\hline 5 & 0.30 & 551.61 & 565.02 & 552.93 & 553.32 & 20.46 & 15.28 & 24.11 & 18.42 \\
\hline 6 & 0.35 & 554.96 & 569.12 & 555.84 & 558.63 & 21.61 & 17.38 & 28.64 & 20.87 \\
\hline 7 & 0.40 & 558.31 & 573.21 & 558.74 & 563.94 & 22.75 & 20.34 & 33.16 & 23.31 \\
\hline 8 & 0.45 & 561.03 & 575.84 & 561.58 & 567.12 & 23.46 & 21.69 & 37.31 & 24.85 \\
\hline 9 & 0.50 & 563.74 & 578.47 & 564.41 & 570.29 & 24.17 & 23,46 & 41.45 & 26.39 \\
\hline 10 & 0.55 & 567.04 & 581.15 & 566.28 & 572.92 & 24.27 & 22.35 & 44.47 & 27.79 \\
\hline 11 & 0.60 & 570.34 & 583.83 & 568.14 & 575.55 & 24.36 & 25.58 & 47.48 & 29.18 \\
\hline 12 & 0.65 & 571.02 & 586.42 & 569.78 & 577.79 & 24.23 & 23.24 & 50.87 & 29.90 \\
\hline 13 & 0.70 & 571.70 & 589.01 & 571.42 & 580.02 & 24.10 & 23.45 & 54.26 & 30.62 \\
\hline 14 & 0.75 & 573.43 & 591.57 & 572.93 & 581.66 & 24.32 & 22.58 & 55.39 & 30.65 \\
\hline 15 & 0.80 & 575.16 & 594.13 & 574.43 & 583.30 & 24.08 & 21.17 & 56.52 & 30.70 \\
\hline 16 & 0.85 & 576.90 & 596.67 & 575.86 & 586.68 & 24.01 & 20.57 & 50.49 & 30.53 \\
\hline 17 & 0.90 & 578.64 & 599.21 & 577.28 & 590.05 & 23.98 & 20.14 & 44.46 & 30.17 \\
\hline
\end{tabular}

a) 动力学参数是从 $\mathrm{TG}$ 曲线上 $464 \sim 594 \mathrm{~K}$ 获得, 其中升温速率为 $15 \mathrm{~K} \mathrm{~min}^{-1}$, b) $\alpha$ 是转化率; c) $T_{\alpha}$ 为转化率 $\alpha$ 所对应的温度; d) $\mathrm{d} \alpha / \mathrm{d} t$ 是两种丝 素膜的转化率 $\alpha$ 的变化速率

规律 $\left(E_{\alpha}\right.$ 值在 $80.00 \sim 250.00 \mathrm{~kJ} \mathrm{~mol}^{-1} ， \ln A$ 在 $16.12 \sim$ $\left.69.08 \mathrm{~s}^{-1}\right)$; (2) 相关系数 $r>0.990 ;$ (3) 由微分和积分两 种方法所得平均 $\ln A$ 值与Kissinger中所测的 $\ln A$ 值最为 接近; (4) 由微分和积分两种方法所得活化能的平均值 与Ozawa法得到的平均活化能最为接近. 如果同时满 足以上 4 个条件, 那么所对应的机理函数即为热分解 反应最可几机理函数 ${ }^{[47]}$.

比较表 11 中的 $E_{\alpha} 、 \ln A$ 和 $r$, 可以看出只有函数序 号为 24 的机理函数同时满足上述 4 个条件, 因此MSF1.5 的热分解反应的机理函数为

$$
f(\alpha)=4(1-\alpha)^{\frac{3}{4}}, g(\alpha)=1-(1-\alpha)^{\frac{1}{4}}
$$

采用与小节3.3.6中所述的相同方法, 计算MSF3.0 在 $464 \sim 594 \mathrm{~K}$ 分解阶段的动力学所对应的动力学参 数, 求得活化能 $E_{\alpha}$ 、指前因子 $\ln A$ 以及相关系数 $r$ (表 12). 通过与上述判定机理函数的 4 点要求相比较, 结果 发现, 也只有 24 号的机理函数最合适. 故其最可几机理 函数仍为: $f(\alpha)=4(1-\alpha)^{\frac{3}{4}}, g(\alpha)=1-(1-\alpha)^{\frac{1}{4}}$

结果表明, 用上述同样方法计算TSF-1.5 和TSF-3.0, 求 得TSF-1.5 的热分解反应的机理函数为: $f(\alpha)=-[\ln (1$ $-\alpha)]^{-1}, g(\alpha)=(1-\alpha) \ln (1-\alpha)+\alpha$; TSF-3.0 的最可几机理函 数仍为: $f(\alpha)=-[\ln (1-\alpha)]^{-1}, g(\alpha)=(1-\alpha) \ln (1-\alpha)+\alpha$. 结果 表明, 两种丝素膜都遵循化学反应过程 ${ }^{[48,49]}$.

\section{4 结论}

本文利用 $1.5 \mathrm{wt} \%$ 和 $3.0 \mathrm{wt} \%$ 浓度的氯化钙-甲酸体 系分别制备了中国丝素膜和泰国丝素膜, 并进行了比 较研究. 结果发现, 氯化钻浓度对两种丝素膜有着相 同的影响机制, 较低氯化钲浓度 $(1.5 \mathrm{wt} \%)$ 体系下制备 的丝蛋白膜主要呈现 $\beta$-折叠结构; 氯化钻浓度增大到 $3.0 \mathrm{wt} \%$ ，膜中丝蛋白以无规线团或 $\alpha$-螺旋结构为主; 在相同浓度制备下得到的两种丝蛋白膜中, MSF的 $\beta$ 折叠含量略低于TSF. 
表 11 MSF-1.5 的热分解微分法和积分法计算结果 ${ }^{\mathrm{a}}$

Table 11 Results of MSF-1.5 thermal decomposition in differential method and integral method ${ }^{\text {a) }}$

\begin{tabular}{|c|c|c|c|c|c|c|c|c|}
\hline \multirow{2}{*}{ 编号 } & \multicolumn{4}{|c|}{ Achar法 } & \multicolumn{4}{|c|}{ Coats-Redfern法 } \\
\hline & $f(x)^{\mathrm{a})}$ & $E_{\alpha}\left(\mathrm{kJ} \mathrm{mol}^{-1}\right)$ & $\ln A\left(\mathrm{~s}^{-1}\right)$ & $r^{\mathrm{c})}$ & $g(x)^{\mathrm{b})}$ & $E_{\alpha}\left(\mathrm{kJ} \mathrm{mol}^{-1}\right)$ & $\ln A\left(\mathrm{~s}^{-1}\right)$ & $r^{c)}$ \\
\hline 1 & $-[\ln (1-\alpha)]^{-1}$ & -2.06 & 2.11 & -0.071 & $\alpha^{\frac{1}{4}}$ & 23.848 & -4.00 & 0.995 \\
\hline 2 & $3 \alpha^{\frac{2}{3}}$ & 8.94 & 4.68 & 0.292 & $\alpha^{\frac{1}{3}}$ & 34.85 & -1.33 & 0.996 \\
\hline 3 & $2 \alpha^{\frac{1}{2}}$ & 30.94 & 9.67 & 0.7184 & $a^{\frac{1}{2}}$ & 56.85 & 3.74 & 0.997 \\
\hline 4 & 1 & 65.47 & 17.11 & 0.631 & $\alpha$ & 122.85 & 18.24 & 0.997 \\
\hline 5 & $\frac{2}{3} \alpha^{-\frac{1}{2}}$ & 162.95 & 38.23 & 0.978 & $\alpha^{\frac{2}{3}}$ & 78.85 & 8.64 & 0.997 \\
\hline 6 & $\alpha$ & -6.57 & 2.67 & -0.175 & $\ln \alpha$ & - & - & - \\
\hline 7 & $\frac{3}{2}(1-\alpha)[-\ln (1-\alpha)]^{1 / 3}$ & 135.97 & 33.14 & 0.991 & {$[-\ln (1-\alpha)]^{\frac{2}{3}}$} & 104.99 & 14.82 & 0.992 \\
\hline 8 & $2(1-\alpha)[-\ln (1-\alpha)]^{1 / 2}$ & 112.71 & 27.92 & 0.992 & {$[-\ln (1-\alpha)]^{\frac{1}{2}}$} & 76.46 & 8.46 & 0.991 \\
\hline 9 & $3(1-\alpha)[-\ln (1-\alpha)]^{\frac{2}{3}}$ & 89.45 & 22.58 & 0.992 & {$[-\ln (1-\alpha)]^{\frac{1}{3}}$} & 47.92 & 1.94 & 0.990 \\
\hline 10 & $4(1-\alpha)[-\ln (1-\alpha)]^{\frac{3}{4}}$ & 77.82 & 19.83 & 0.992 & {$[-\ln (1-\alpha)]^{\frac{1}{4}}$} & 33.65 & -1.44 & 0.989 \\
\hline 11 & $\alpha(1-\alpha)$ & 78.97 & 21.98 & 0.957 & $\ln \left(\frac{\alpha}{1-\alpha}\right)$ & 737.10 & 149.23 & 0.964 \\
\hline 12 & $\frac{1}{2}(1-\alpha)[-\ln (1-\alpha)]^{-1}$ & 322.03 & 73.67 & 0.987 & {$[-\ln (1-\alpha)]^{2}$} & 333.28 & 64.383 & 0.992 \\
\hline 13 & $\frac{1}{3}(1-\alpha)[-\ln (1-\alpha)]^{-2}$ & 461.58 & 103.65 & 0.985 & {$[-\ln (1-\alpha)]^{3}$} & 504.5 & 101.10 & 0.992 \\
\hline 14 & $\frac{1}{4}(1-\alpha)[-\ln (1-\alpha)]^{-3}$ & 601.13 & 133.52 & 0.984 & {$[-\ln (1-\alpha)]^{4}$} & 675.72 & 137.71 & 0.993 \\
\hline 15 & $2(1-\alpha)^{\frac{1}{2}}$ & 139.71 & 33.05 & 0.999 & $1-(1-\alpha)^{\frac{1}{2}}$ & 140.61 & 21.69 & 0.998 \\
\hline 16 & $3(1-\alpha)^{\frac{2}{3}}$ & 153.97 & 35.87 & 0.999 & $1-(1-\alpha)^{\frac{1}{3}}$ & 147.33 & 22.85 & 0.997 \\
\hline 17 & $\frac{1}{2} \alpha-1$ & - & - & - & $\alpha^{2}$ & 254.86 & 46.43 & 0.998 \\
\hline 18 & $-[\ln (1-\alpha)]^{-1}$ & 236.49 & 53.67 & 0.999 & $(1-\alpha) \ln (1-\alpha)+\alpha$ & 276.52 & 50.715 & 0.999 \\
\hline 19 & $\frac{3}{2}\left[1-(1-\alpha)^{\frac{1}{3}}\right]^{-1}(1-\alpha)^{\frac{2}{3}}$ & 280.03 & 61.99 & 0.996 & {$\left[1-(1-\alpha)^{\frac{1}{3}}\right]^{2}$} & 303.82 & 55.458 & 0.997 \\
\hline 20 & $\frac{3}{2}\left[1-(1-\alpha)^{\frac{1}{3}}\right]^{-1}$ & 223.01 & 49.12 & 0.997 & $\left(1-\frac{2}{3} \alpha\right)-(1-\alpha)^{\frac{2}{3}}$ & 285.49 & 51.265 & 0.998 \\
\hline 21 & $1-\alpha$ & 182.48 & 43.40 & 0.989 & $-\ln (1-\alpha)$ & 162.07 & 27.36 & 0.992 \\
\hline 22 & $(1-\alpha)^{2}$ & 268.02 & 62.71 & 0.953 & $1 /(1-\alpha)$ & 84.48 & 11.48 & 0.788 \\
\hline 23 & $1(1-\alpha)^{3}$ & 353.55 & 82.71 & 0.926 & {$\left[\frac{1}{1-\alpha}\right]^{2}$} & 178.12 & 33.29 & 0.804 \\
\hline 24 & $4(1-\alpha)^{\frac{3}{4}}$ & 161.10 & 37.19 & 0.997 & $1-(1-\alpha)^{\frac{1}{4}}$ & 150.85 & 23.38 & 0.996 \\
\hline 25 & $\frac{1}{2}(1-\alpha)^{-\frac{2}{3}}$ & 39.92 & 11.91 & 0.489 & $(1-\alpha)^{-\frac{1}{2}}$ & 37.66 & 0.13 & 0.753 \\
\hline
\end{tabular}


(续表11)

\begin{tabular}{lcccccccc}
26 & $\left.\frac{3}{2}(1-\alpha)^{\frac{4}{3}}(1-\alpha)^{-\frac{1}{3}}-1\right]^{-1}$ & 365.57 & 81.30 & 0.975 & {$\left[(1-\alpha)^{-\frac{1}{3}}-1\right]^{2}$} & 384.01 & 74.55 & 0.980 \\
27 & $6(1-\alpha)^{\frac{2}{3}}\left[1-(1-\alpha)^{\frac{1}{3}}\right]^{\frac{1}{2}}$ & 90.94 & 22.46 & 0.998 & {$\left[1-(1-\alpha)^{\frac{1}{3}}\right]^{\frac{1}{2}}$} & 69.09 & 6.15 & 0.996 \\
28 & $4\left\{(1-\alpha)\left[1-(1-\alpha)^{\frac{1}{2}}\right]\right\}^{\frac{1}{2}}$ & 122.54 & 29.79 & 0.986 & {$\left[1-(1-\alpha)^{\frac{1}{2}}\right]^{\frac{1}{2}}$} & 65.73 & 5.54 & 0.998 \\
29 & $\frac{1}{2}(1-\alpha)^{-1}$ & 11.41 & 5.47 & 0.125 & $1-(1-\alpha)^{2}$ & 96.24 & 12.66 & 0.983 \\
30 & $\frac{1}{3}(1-\alpha)^{-2}$ & -74.12 & -13.43 & -0.444 & $1-(1-\alpha)^{3}$ & 77.78 & 8.64 & 0.961 \\
31 & $\frac{1}{4}(1-\alpha)^{-3}$ & -159.66 & -32.45 & -0.608 & $1-(1-\alpha)^{4}$ & 64.36 & 5.65 & 0.937 \\
\hline
\end{tabular}

a) 动力学参数是从 $\mathrm{TG}$ 曲线上 $464 \sim 594 \mathrm{~K}$ 获得, 其中升温速率为 $15 \mathrm{~K} \mathrm{~min}^{-1}$; b) $f(x)$ 为微分形式机理函数; c) $g(x)$ 为积分形式机理函数, d) $r$ 为相关系数

表 12 MSF-3.0的热分解微分法和积分法计算结果 ${ }^{\text {a) }}$

Table 12 Results of MSF-1.5 thermal decomposition in differential method and integral method ${ }^{\text {a) }}$

\begin{tabular}{|c|c|c|c|c|c|c|c|c|}
\hline \multirow{2}{*}{ 编号 } & \multicolumn{4}{|c|}{ Achar法 } & \multicolumn{4}{|c|}{ Coats-Redfern法 } \\
\hline & $f(x)^{\mathrm{b})}$ & $E_{\alpha}\left(\mathrm{kJ} \mathrm{mol}^{-1}\right)$ & $\ln A\left(\mathrm{~s}^{-1}\right)$ & $r^{\mathrm{d})}$ & $g(x)^{\mathrm{c})}$ & $E_{\alpha}\left(\mathrm{kJ} \mathrm{mol}^{-1}\right)$ & $\ln A\left(\mathrm{~s}^{-1}\right)$ & $r^{\mathrm{d})}$ \\
\hline 1 & $4 \alpha^{\frac{3}{4}}$ & 16.75 & 6.32 & 0.646 & $\alpha^{\frac{1}{4}}$ & 21.50 & -4.59 & 0.880 \\
\hline 2 & $3 a^{\frac{2}{3}}$ & 26.94 & 8.73 & 0.808 & $\alpha^{\frac{1}{3}}$ & 31.69 & -2.08 & 0.925 \\
\hline 3 & $2 \alpha^{\frac{1}{2}}$ & 47.33 & 13.37 & 0.925 & $\alpha^{\frac{1}{2}}$ & 52.08 & 2.65 & 0.964 \\
\hline 4 & 1 & 82.27 & 20.92 & 0.790 & $\alpha$ & 113.24 & 16.1402 & 0.987 \\
\hline 5 & $\frac{2}{3} \alpha^{-\frac{1}{2}}$ & 169.64 & 39.90 & 0.994 & $\alpha^{\frac{2}{3}}$ & 72.46 & 7.22 & 0.989 \\
\hline 6 & $\alpha$ & -13.83 & 1.35 & 0.560 & $\ln \alpha$ & - & - & - \\
\hline 7 & $\frac{3}{2}(1-\alpha)[-\ln (1-\alpha)]^{1 / 3}$ & 136.92 & 33.61 & 0.958 & {$[-\ln (1-\alpha)]^{\frac{2}{3}}$} & 95.03 & 12.63 & 0.987 \\
\hline 8 & $2(1-\alpha)[-\ln (1-\alpha)]^{1 / 2}$ & 110.89 & 27.80 & 0.95 & {$[-\ln (1-\alpha)]^{\frac{1}{2}}$} & 69.01 & 6.78 & 0.985 \\
\hline 9 & $3(1-\alpha)[-\ln (1-\alpha)]^{\frac{2}{3}}$ & 84.86 & 21.87 & 0.936 & {$[-\ln (1-\alpha)]^{\frac{1}{3}}$} & 42.98 & 0.79 & 0.978 \\
\hline 10 & $4(1-\alpha)[-\ln (1-\alpha)]^{\frac{3}{4}}$ & 71.85 & 18.82 & 0.925 & {$[-\ln (1-\alpha)]^{\frac{1}{4}}$} & 29.96 & -2.33 & 0.971 \\
\hline 11 & $\alpha(1-\alpha)$ & 66.66 & 19.63 & 0.817 & $\ln \left(\frac{\alpha}{1-\alpha}\right)$ & 791.63 & 161.02 & 0.951 \\
\hline 12 & $\frac{1}{2}(1-\alpha)[-\ln (1-\alpha)]^{-1}$ & 345.14 & 78.88 & 0.975 & {$[-\ln (1-\alpha)]^{2}$} & 303.26 & 57.96 & 0.984 \\
\hline 13 & $\frac{1}{3}(1-\alpha)[-\ln (1-\alpha)]^{-2}$ & 501.31 & 112.42 & 0.978 & {$[-\ln (1-\alpha)]^{3}$} & 459.42 & 91.51 & 0.982 \\
\hline 14 & $\frac{1}{4}(1-\alpha)[-\ln (1-\alpha)]^{-3}$ & 657.48 & 145.84 & 0.981 & {$[-\ln (1-\alpha)]^{4}$} & 615.59 & 124.93 & 0.981 \\
\hline 15 & $2(1-\alpha)^{\frac{1}{2}}$ & 148.73 & 35.22 & 0.992 & $1-(1-\alpha)^{\frac{1}{2}}$ & 128.61 & 19.08 & 0.988 \\
\hline
\end{tabular}




\begin{tabular}{|c|c|c|c|c|c|c|c|c|}
\hline & & & & & & & & (续表12) \\
\hline 16 & $3(1-\alpha)^{\frac{2}{3}}$ & 162.14 & 37.86 & 0.984 & $1-(1-\alpha)^{\frac{1}{3}}$ & 134.41 & 20.04 & 0.988 \\
\hline 17 & $\frac{1}{2} \alpha-1$ & - & - & - & $\alpha^{2}$ & 235.55 & 42.30 & 0.988 \\
\hline 18 & $-[\ln (1-\alpha)]^{-1}$ & 264.65 & 59.91 & 0.997 & $(1-\alpha) \ln (1-\alpha)+\alpha$ & 254.35 & 45.97 & 0.988 \\
\hline 19 & $\frac{3}{2}\left[1-(1-\alpha)^{\frac{1}{3}}\right]^{-1}(1-\alpha)^{\frac{2}{3}}$ & 305.63 & 67.71 & 0.988 & {$\left[1-(1-\alpha)^{\frac{1}{3}}\right]^{2}$} & 277.89 & 49.91 & 0.986 \\
\hline 20 & $\frac{3}{2}\left[1-(1-\alpha)^{\frac{1}{3}}\right]^{-1}$ & 251.97 & 55.52 & 0.998 & $\left(1-\frac{2}{3} \alpha\right)-(1-\alpha)^{\frac{2}{3}}$ & 262.09 & 46.26 & 0.988 \\
\hline 21 & $1-\alpha$ & 188.97 & 45.06 & 0.966 & $-\ln (1-\alpha)$ & 147.09 & 24.11 & 0.987 \\
\hline 22 & $(1-\alpha)^{2}$ & 269.46 & 63.34 & 0.919 & $1 /(1-\alpha)$ & 71.41 & 8.53 & 0.982 \\
\hline 23 & $1(1-\alpha)^{3}$ & 349.95 & 82.31 & 0.889 & {$\left[\frac{1}{1-\alpha}\right]^{2}$} & 151.9 & 27.57 & 0.976 \\
\hline 24 & $4(1-\alpha)^{\frac{3}{4}}$ & 168.85 & 39.10 & 0.980 & $1-(1-\alpha)^{\frac{1}{4}}$ & 137.44 & 20.46 & 0.988 \\
\hline 25 & $\frac{1}{2}(1-\alpha)^{-\frac{2}{3}}$ & 54.83 & 15.28 & 0.670 & $(1-\alpha)^{-\frac{1}{2}}$ & 31.17 & -1.44 & 0.978 \\
\hline 26 & $\frac{3}{2}(1-\alpha)^{\frac{4}{3}}\left[(1-\alpha)^{-\frac{1}{3}}-1\right]^{-1}$ & 386.12 & 85.99 & 0.961 & {$\left[(1-\alpha)^{-\frac{1}{3}}-1\right]^{2}$} & 346.78 & 66.59 & 0.982 \\
\hline 27 & $6(1-\alpha)^{\frac{2}{3}}\left[1-(1-\alpha)^{\frac{1}{3}}\right]^{\frac{1}{2}}$ & 90.402 & 22.60 & 0.976 & {$\left[1-(1-\alpha)^{\frac{1}{3}}\right]^{\frac{1}{2}}$} & 62.66 & 4.70 & 0.982 \\
\hline 28 & $4\left\{(1-\alpha)\left[1-(1-\alpha)^{\frac{1}{2}}\right]\right\}^{\frac{1}{2}}$ & 120.13 & 29.55 & 0.940 & {$\left[1-(1-\alpha)^{\frac{1}{2}}\right]^{\frac{1}{2}}$} & 59.76 & 4.19 & 0.986 \\
\hline 29 & $\frac{1}{2}(1-\alpha)^{-1}$ & 28.00 & 9.19 & 0.322 & $1-(1-\alpha)^{2}$ & 90.01 & 11.29 & 0.968 \\
\hline 30 & $\frac{1}{3}(1-\alpha)^{-2}$ & -52.49 & -8.69 & 0.341 & $1-(1-\alpha)^{3}$ & 73.69 & 7.73 & 0.882 \\
\hline 31 & $\frac{1}{4}(1-\alpha)^{-3}$ & -133.00 & -26.68 & 0.531 & $1-(1-\alpha)^{4}$ & 61.69 & 5.06 & 0.603 \\
\hline
\end{tabular}

a) 动力学参数是从 $\mathrm{TG}$ 曲线上 $464 \sim 594 \mathrm{~K}$ 获得, 其中升温速率为 $15 \mathrm{~K} \mathrm{~min}^{-1}$; b) $f(x)$ 为微分形式机理函数; c) $g(x)$ 为积分形式机理函数; d) $r$ 为相关系数

分别运用Kissinger、Ozawa和Vyazovkin方法进行 线性回归拟合求解其活化能发现, 在464 594 K温度区 间，这三种方法求得的活化能相差不大. MSF-1.5和 M S F - 3 .0 的平均活化能分别为 157.92 和 $154.89 \mathrm{~kJ} \mathrm{~mol}^{-1}$. TSF-1.5 和TSF-3.0分别为 189.89 和 $174.52 \mathrm{~kJ} \mathrm{~mol}^{-1}$; 根据DTG曲线上最大的峰值温度计 算出了热力学参数 $\left(\Delta H^{\neq} 、 \Delta G^{\neq} 、 \Delta S^{\neq}\right) . \Delta G^{\neq}>0$ 表明, MSF-3.0不同升温速率所对应的 $\Delta G^{\neq}$和 $\Delta H^{\neq}$的值都比
MSF-1.5的要小, 可见MSF-3.0更容易发生热分解行为. TSF呈现相同的情况. 此外, 通过等温失重时间计算发 现, 在 $464 \mathrm{~K}$ 下, MSF-3.0失重 $0.50 \%$ 需要 $5.2 \mathrm{~s}$ 左右, 而 MSF-1.5大约需要 $13.5 \mathrm{~s}$, 说明MSF-1.5较MSF-3.0有更 好的热稳定性. TSF 呈现相同性质. 在相同浓度体系下 制备得到的丝素膜, 泰国丝素膜具有更好的热稳定性. 另外, 中国丝素膜和泰国丝素膜的热分解机理都遵循 二维扩散机制, 符合Valensi方程.

\section{参考文献}

1 Ha AW, Kang HJ, Kim SL, Kim MH, Kim WK. Prev Nutr Food Sci, 2018, 23: 70-76 
2 Yu S, Yang W, Chen S, Chen M, Liu Y, Shao Z, Chen X. RSC Adv, 2014, 4: 18171-18177

3 Chen M, Shao Z, Chen X. J Biomed Mater Res, 2012, 100A: 203-210

4 Koh LD, Cheng Y, Teng CP, Khin YW, Loh XJ, Tee SY, Low M, Ye E, Yu HD, Zhang YW, Han MY. Prog Polym Sci, 2015, 46: 86-110

5 Numata K, Ifuku N, Masunaga H, Hikima T, Sakai T. Biomacromolecules, 2017, 18: 1937-1946

6 Byette F, Bouchard F, Pellerin C, Paquin J, Marcotte I, Mateescu MA. Polym Bull, 2011, 67: 159-175

7 Zhou J, Zhang B, Shi L, Zhong J, Zhu J, Yan J, Wang P, Cao C, He D. ACS Appl Mater Interfaces, 2014, 6: 21813-21821

8 Nultsch K, Germershaus O. Eur J Pharm Sci, 2017, 106: 254-261

9 Zhang F, Lu Q, Yue X, Zuo B, Qin M, Li F, Kaplan DL, Zhang X. Acta Biomater, 2014, 12: 139-145

10 Matsumoto K, Uejima H, Iwasaki T, Sano Y, Sumino H. J Appl Polym Sci, 1996, 60: 503-511

11 Zhang C, Song D, Lu Q, Hu X, Kaplan DL, Zhu H. Biomacromolecules, 2012, 13: 2148-2153

12 Lu Q, Hu X, Wang X, Kluge JA, Lu S, Cebe P, Kaplan DL. Acta Biomater, 2010, 6: 1380-1387

13 Rockwood DN, Preda RC, Yücel T, Wang X, Lovett ML, Kaplan DL. Nat Protoc, 2011, 6: 1612-1631

14 Wang F, Wolf N, Rocks EM, Vuong T, Hu X. J Therm Anal Calorim, 2015, 122: 1069-1076

15 Wang F, Yu H, Gu ZG, Si L, Liu Q, Hu X. J Therm Anal Calorim, 2017, 130: 851-859

16 Wang F, Chandler P, Oszust R, Sowell E, Graham Z, Ardito W, Hu X. J Therm Anal Calorim, 2017, 127: 923-929

17 Liu Q, Wang F, Gu Z, Ma Q, Hu X. Int J Mol Sci, 2018, 19: 3309

\section{1-2292]}

38 Fernandez A, Saffe A, Pereyra R, Mazza G, Rodriguez R. Appl Thermal Eng, 2016, 106: 1157-1164

39 Tao H, Kaplan DL, Omenetto FG. Adv Mater, 2012, 24: 2824-2837

40 Chaiyo N, Muanghlua R, Niemcharoen S, Boonchom B, Seeharaj P, Vittayakorn N. J Therm Anal Calorim, 2012, 107: 1023-1029

41 Kandelbauer A, Wuzella G, Mahendran A, Taudes I, Widsten P. Chem Eng J, 2009, 152: 556-565

42 Zhuo L, Kou K, Yao P, Wu G, Wang Y. J Therm Anal Calorim, 2015, 119: 2039-2051

43 Guo H, Wang Y, Ni X. J China Univ Min Technol, 2016, 45: 591-596 (in Chinese) [郭慧, 王延斌, 倪小明. 中国矿业大学学报, 2016, 45: 591596]

44 Liu W, Lin M. Guangdong Chem Ind, 2004, 31: 6-8 (in Chinese) [刘文峰, 林木良. 广东化工, 2004, 31: 6-8] 


\title{
Comparative studies of structure, thermal decomposition mechanism and thermodynamic parameters of two kinds of silk fibroin films
}

\author{
Qichun Liu ${ }^{1,2}$, Fang Wang ${ }^{1 *}$, Yingying $\mathrm{Li}^{1,2}$, Haiyang $\mathrm{Yu}^{1,2}$, Qingyu $\mathrm{Ma}^{3}$, Zhenggui $\mathrm{Gu}^{2 *}$ \\ ${ }^{1}$ Center of Analysis and Testing, Nanjing Normal University, Nanjing 210023, China \\ ${ }^{2}$ School of Chemistry and Materials Science, Nanjing Normal University, Nanjing 210023, China \\ ${ }^{3}$ School of Physics and Technology, Nanjing Normal University, Nanjing 210023, China \\ *Corresponding authors (email: wangfang@njnu.edu.cn; guzhenggui@njnu.edu.cn)
}

\begin{abstract}
The silk fibroin films from Chinese and Thai silkworms were prepared by a new method of calcium chlorideformic acid system. The films were characterized by scanning electron microscopy, Fourier transform infrared spectroscopy, X-ray diffraction and thermogravimetric analysis. The morphological, molecular conformation and thermal stability of the material and the thermal decomposition mechanism were obtained as well as the kinetic parameters (activation energy $E$, pre-exponential factor $A$ ) and thermodynamic parameters $\left(\Delta H^{\ddagger}, \Delta G^{\ddagger}\right.$ and $\Delta S^{\ddagger}$ ), and a compare research were performed. The results showed that the structure of silk fibroin film under $1.5 \mathrm{wt} \%$ concentration system mainly appeared $\beta$-sheet, and the membrane structure under $3.0 \mathrm{wt} \%$ concentration system was dominated by random coils or $\alpha$-helix; At the different heating rate, the decomposition temperature, activation energy and activation enthalpy of $3.0 \mathrm{wt} \%$ concentration system were all lower than those of $1.5 \mathrm{wt} \%$, and the latter has better thermal stability than the former. Under the same concentration, the $\beta$-sheet content of Thai silk fibroin film is slightly higher than that of China and it had better thermal stability. It was found by Achar differential method and Coats-Redfern integration method that the thermal decomposition process of silk fibroin film follows the cylindrical symmetry (two-dimensional diffusion) mechanism at 464 594 K.
\end{abstract}

Keywords: silk fibroin, molecular conformation, thermal decomposition mechanism, thermodynamics, thermal analysis

doi: $10.1360 / \mathrm{N} 032018-00265$ 